\title{
Numerical simulation of the interaction of two shear layers in double backward-facing steps
}

Cite as: Phys. Fluids 31, 056106 (2019); https://doi.org/10.1063/1.5083986

Submitted: 03 December 2018 . Accepted: 07 May 2019 . Published Online: 29 May 2019

Fang Deng (邓放) (D), Guilai Han (韩桂来) (D, Meikuan Liu (刘美宽) (D), Jue Ding (丁珏), Peifen Weng (翁培 奋), and Zonglin Jiang (姜宗林)

\section{ARTICLES YOU MAY BE INTERESTED IN}

Dynamic mode decomposition with exogenous input for data-driven modeling of unsteady flows

Physics of Fluids 31, 057106 (2019); https://doi.org/10.1063/1.5093507

Fast flow field prediction over airfoils using deep learning approach

Physics of Fluids 31, 057103 (2019); https://doi.org/10.1063/1.5094943

Numerical investigation of highly unsteady accelerated/decelerated flows for blunt bodies experiencing impulsive motion

Physics of Fluids 31, 055108 (2019); https://doi.org/10.1063/1.5089674

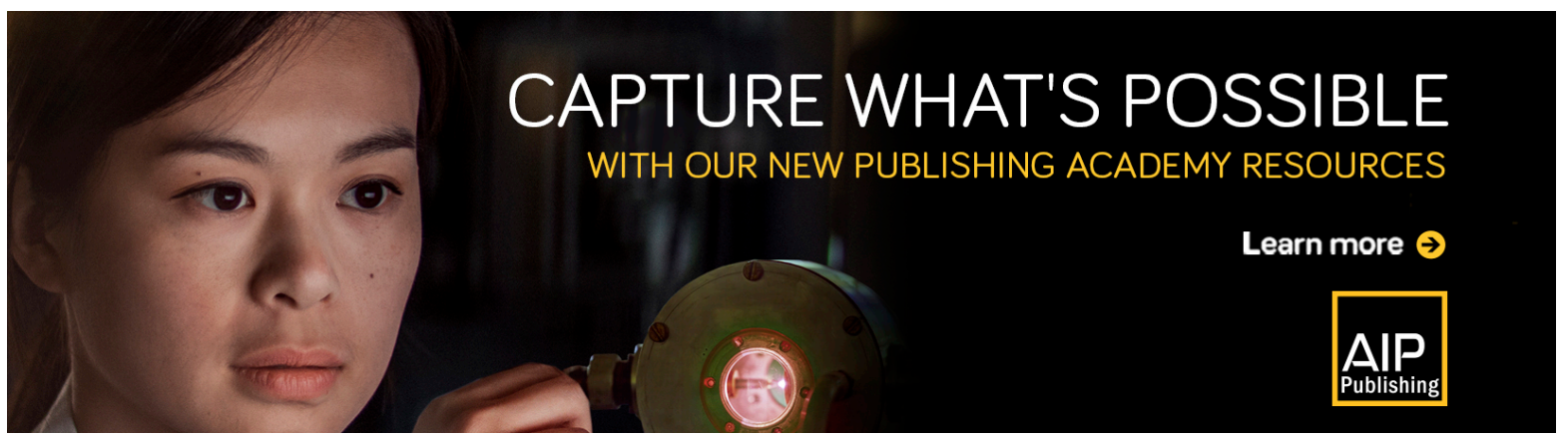




\title{
Numerical simulation of the interaction of two shear layers in double backward-facing steps
}

\author{
Cite as: Phys. Fluids 31, 056106 (2019); doi: 10.1063/1.5083986 \\ Submitted: 3 December 2018 - Accepted: 7 May 2019 • \\ Published Online: 29 May 2019
}

\author{
Fang Deng (邓放), ${ }^{1,2,3}$ (D) Guilai Han (韩桂来), ${ }^{1,3}$ (D) Meikuan Liu (刘美宽), ${ }^{1,3}$ (D) Jue Ding (丁珏), ${ }^{2}$ \\ Peifen Weng (翁培奋), ${ }^{2}$ and Zonglin Jiang (姜宗林) ${ }^{1,3}$
}

\author{
AFFILIATIONS \\ 'State Key Laboratory of High Temperature Gas Dynamics, Institute of Mechanics, Chinese Academy of Sciences, \\ Beijing 100190, China \\ ${ }^{2}$ Shanghai Institute of Applied Mathematics and Mechanics, Shanghai University, Shanghai 200072, China \\ ${ }^{3}$ School of Engineering Science, University of Chinese Academy of Sciences, Beijing 100049, China
}

\begin{abstract}
In this paper, Navier-Stokes equations were solved with high-order accurate schemes to investigate the basic structure and regularity of the flow field during the interaction of a supersonic jet and a codirectional supersonic incoming flow. A double backward-facing step model was proposed to investigate the interaction between the jet/supersonic incoming flow shear layers. The two shear layers interact to produce a secondary jet. The secondary jet produced by the action has a unique periodicity that is related to the overall oscillation of the shear layer. The secondary jet is generated when the horizontal angle of the jet shear layer reaches a certain value. This paper focused on the analysis and discussion of the periodicity of the secondary jet. When the aspect ratio is different, the period of the secondary jet changes significantly. However, when the static pressure ratio is different, the period of the secondary jet does not change much.
\end{abstract}

Published under license by AIP Publishing. https://doi.org/10.1063/1.5083986

\section{INTRODUCTION}

The interaction between the jet and supersonic incoming flow is an important flow phenomenon in aerodynamics. For example, in a typical dual-mode scramjet engine, a fuel injection strut is used in the combustion chamber to improve the mixing of the fuel with the supersonic airflow. ${ }^{1}$ During a rocket launch, a supersonic jet is formed in the nozzle tail and interacts with the airflow around the rocket body; different types of jet flows are formed with increasing height. ${ }^{2-4}$ During the flight of a supersonic cruise missile, the jet formed by the engine nozzle interacts with the airflow around the projectile. ${ }^{5}$ Therefore, the interaction of the jet and supersonic flow is very important from a practical engineering perspective and for scientific research.

To date, studies of the shear layer have mainly focused on the use of models of the cavity and the backward-facing steps. Ma and Schröder ${ }^{6}$ conducted an experimental investigation on the flapping motion of the turbulent reattaching shear layer downstream of a backward-facing step. The purpose of their study was to analyze the flapping motion, which is characterized by the instability of the reattached shear layer and its interaction with the recirculation zone. The maximum reverse flow and the unsteady motion of the reconnection point are correlated with the tapping motion. Therefore, in the latter half of the reattachment length, the flapping motion contributes most of the Reynolds shear stress and the turbulent kinetic energy within the shear layer. Park and Jeon ${ }^{7}$ used tabs located at the edge of the backward-facing step to investigate mixing enhancement. By creating counter-rotating flow vortices and bringing the high momentum fluid into the recirculation zone, the tabs introduced a strong disturbance to the separation shear layer. Velocity fluctuations in the shear layer increased almost everywhere, and these fluctuations continue until downstream. As a result, by increasing the entrained high momentum fluid entering the recirculation zone, the reattachment length at all spanwise position locations was reduced. In experiments, Kegerise et al. ${ }^{8}$ discovered largescale entrapped vortex structures with supersonic shear layers in an unstable mode. It was considered that the modes coexisted or that mode switching led to the pressure fluctuation inside the cavity; therefore, several peaks occurred in the power spectral density and it was concluded that a strong correlation existed between the 
dominant Rossiter mode and the cavity shear-layer structure. Sheu and Rani ${ }^{9}$ investigated eddy structures and their interactions in a three-dimensional channel with a backward-facing step numerically. Strong interactions between shear layer instability provided clear indications. The simulation provides evidence that the strong sensitivity of the flow in the recirculation zone was a specific change in the evolution of the vortex structure within the separated internal shear layer due to disturbance. The event could enhance the flapping motion of the inner shear layer. It was found that the Kelvin-Helmholtz-like instability developed near the step wall interface caused the rolling up of the downstream shear layer. Hall et al. ${ }^{10}$ investigated the vortex structures and the shear layer behind the step. The main vortex-shear interaction may be the most important in the backward-facing step flow. The shear layer provides additional mass and momentum to the primary vortex through viscosity, turbulent mixing, and mass transfer. In contrast, the low pressure created by the primary eddy current is responsible for the downward bending of the shear layer.

Research on shear layers mainly focus on the investigation of the interaction between the shear layer and the shock wave/expansion wave and the shear layer and the boundary layer. However, there are no suitable models to study the interaction between two different shear layers. Génin and Menon ${ }^{11}$ studied the interaction between two oblique shocks and a turbulent shear layer. Research indicated that the turbulence evolution is mainly affected by two competing phenomena. The interaction of the shear layer and the shock wave causes a scattering of the closure coefficients at the edge of the mixing layer with less turbulent motion. However, their behavior has not been strongly modified within the layer thickness. Suzuki and Lele ${ }^{12}$ used the geometric acoustic theory and direct numerical simulation (DNS) to investigate the interaction between a two-dimensional supersonic shear layer and an expansion wave. It was found that the compressible wave occurred at the saddle point between the shear layer vortices where leakage occurred. Cohen and Bennett ${ }^{13}$ used a laser tester to measure the velocity field of the backward-facing step perturbed by the pulsation of the incoming gas flow. The interaction between the shear layer and the boundary layer was determined, and the results showed that changes in the disturbance frequency resulted in periodic increases and decreases in the recirculation zone downstream of the step and the flow behavior deviated significantly from a quasisteady performance.

There have also been a few studies recently focusing on the phenomena between two shear layers. Weidman and Wang ${ }^{14}$ presented solutions for the boundary layer between two shear flows of different strengths in the same direction. Similarity transformations reduced the boundary-layer equations to a pair of ordinary differential equations governed by three dimensionless parameters: the shear strength ratio, the density ratio, and the viscosity ratio. The solution to the original boundary value problem showed consistency with the initial value integration, but using this method could find additional dual and quadruple solutions. They found that these additional solutions are not feasible through physical reasons and disagreement analyses.

In this study, for the first time, we develop a double backwardfacing step model to simulate the interaction between the two shear layers. The interaction between the jet shear layer and supersonic incoming flow shear layer is simulated by using the fifthorder weighted essentially nonoscillatory (WENO) ${ }^{15}$ scheme and the sixth-order central difference discretization ${ }^{16}$ for the convective and viscous terms. The solution in time is advanced with the third-order Runge-Kutta scheme, ${ }^{17}$ and the parallel computation is performed by using the message passing interface (MPI) for the nonblocking communication.

\section{THEORETICAL APPROACH}

\section{A. The physical model}

The physical model of the jet and codirectional supersonic incoming flow is shown in Fig. 1(a). In this study, a double backward-facing step model is developed; it consists of two steps that were calibrated as shown in Fig. 1(a). Above the wall BC, supersonic incoming flow occurs; when the supersonic incoming flow flows through the back steps, a shear layer is formed at the corner of the backward-facing step, and this layer is called the supersonic incoming flow shear layer. The jet flow originates from the left end of the second step. Above the jet, another shear layer is created and is called the jet shear layer. The area between the supersonic incoming flow shear layer and the jet shear layer is called the mixing zone. The interaction between the two shear layers causes a complex phenomenon in the mixing zone; the details will be explained in Secs. III A-III D.

The experimental investigation will be conducted in supersonic and hypersonic tunnels by cooperating with other teams. The tunnels with rectangular nozzle exits were chosen for the experiments and cooperation. The experimental model was designed as a rectangular exit for the jet after the backward-facing step. Hence, the threedimensional effects can be weakened in the experiments, and the two-dimensional Navier-Stokes equation was adopted as the governing equation for such a preliminary investigation. By weakening the three-dimensional effects, the investigations on the formation and evolution of the strong structures in the flow field can be focused in this paper, including the shear layers, shock waves, and jets, so that factors such as pressure ratio and geometrical shape can be discussed in this paper independently and respectively. In the future, the threedimensional effects will be gradually introduced into numerical investigations by the two-dimensional axisymmetric Navier-Stokes equation for two concentric flows and three-dimensional NavierStokes equations for nonconcentric flows. Meanwhile, the experiments will be carried out for other models with more complex geometrical shapes. Then, the three-dimensional effects might play an important role for the formation and evolution of the structures, beside the factors investigated in this paper. So the two-dimensional Navier-Stokes equation was adopted as the governing equation for this preliminary investigation.

For the primary stage, the strong structures, such as shear layers, jets, secondary jets, and secondary shock waves, were dominated by the large scale of the flow field, rather than small or detailed structures. Comparing with the effect of the strong structures, the effect of small eddies or turbulence for the flow nearby the backward-facing steps might not be significant enough. The evolution and dissipation of the flow in the downstream might be dominated by eddies and turbulence, which is a little far from the interaction between the two shear layers. However, for supersonic or hypersonic flows, the perturbations or disturbances, propagating at sonic speed, are unable to spread to the upstream. Therefore, the interaction between shear 


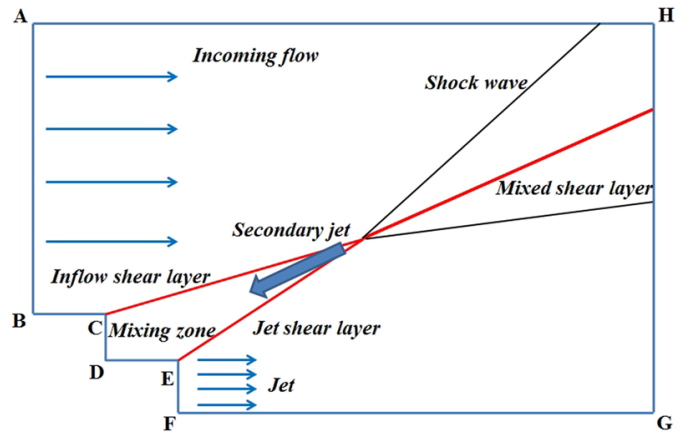

(a)

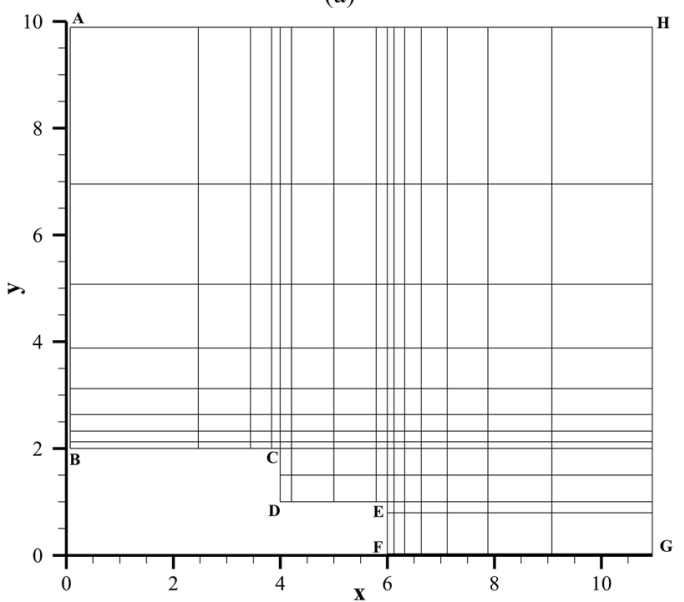

(b)

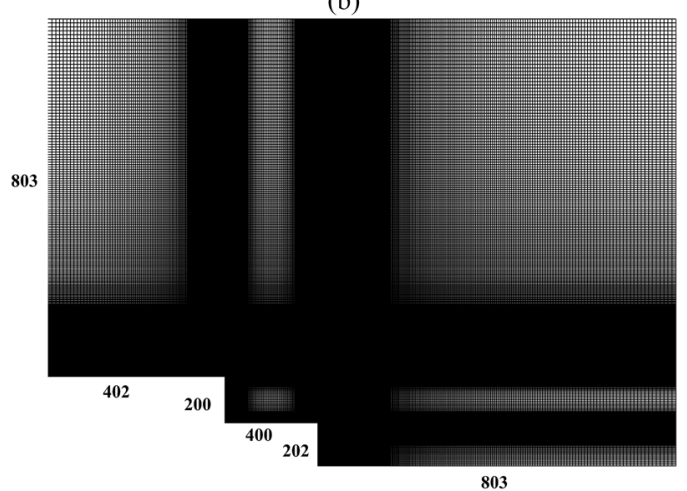

(c)

FIG. 1. The physical model and the calculative region: (a) basic physical structure, (b) calculation domain and CPU division, and (c) the mesh diagram of the flow field.

layers cannot be affected by the flow downstream, even if it is dominated by small eddies or turbulences. So the laminar assumption is used to study the phenomenon of shear layer interaction.

The calculation domain is shown in Fig. 1(b). A double backward-facing step model was used in this numerical simulation. Above the first back step at $\mathrm{BC}$, supersonic incoming flow with $\mathrm{Ma}=3$ occurs. A high-temperature and high-pressure jet with $\mathrm{Ma}=2$ occurs on the right side of the second backward-facing step at EF. When the jet pressure at EF is higher than the ambient pressure, the jet is called an under-expanded jet. When the jet pressure at $\mathrm{EF}$ is lower than the ambient pressure, the jet is called an overexpanded jet. The interaction between the supersonic jet and the codirectional supersonic flow occurs at the second backward facing step and a complex flow field structure is formed. In this study, five different flow conditions were evaluated, and for each condition, the boundary conditions were set as shown in Table I.

$1605 \times 1203$ grids and $168 \mathrm{CPU}$ cores were used running on a supercomputer in Guangzhou (Tianhe II). Figure 1(c) is a schematic diagram of the flow field with grid points marked on each side. The entire flow field used rectangular orthogonal grids. Grid compression arithmetic was used near the wall to ensure the accuracy of the flow field and observe the physical structure well. The bottom grid scale is 0.001 corresponding to the characteristic length EF.

Changes in the ratio of the jet static pressure to the supersonic incoming flow static pressure may result in different flow fields and parameter changes. The parameters used for the five tests, such as density, pressure, $\mathrm{Re}$, and $\mathrm{Ma}$ of the supersonic incoming flow and jet under different static pressure ratios are listed in Tables II and III. The static pressure ratios are $0.6,1.2,2.5,10$, and 30 . The component of the supersonic incoming flow and the jet flow is air.

\section{B. Governing equations}

In this study, the nondimensional compressible Navier-Stokes equation is used as the governing equation,

$$
\frac{\partial \mathbf{U}}{\partial t}+\frac{\partial \mathbf{F}}{\partial x}+\frac{\partial \mathbf{G}}{\partial y}=\frac{1}{\operatorname{Re}}\left(\frac{\partial \mathbf{F}_{v}}{\partial x}+\frac{\partial \mathbf{G}_{v}}{\partial y}\right) .
$$

The vector forms are as follows:

$$
\begin{gathered}
\mathbf{U}=\left[\begin{array}{c}
\rho \\
\rho u \\
\rho v \\
E
\end{array}\right], \mathbf{F}=\left[\begin{array}{c}
\rho u \\
\rho u^{2}+p \\
\rho u v \\
(E+p) u
\end{array}\right], \mathbf{G}=\left[\begin{array}{c}
\rho v \\
\rho u v \\
\rho v^{2}+p \\
(E+p) v
\end{array}\right], \\
\mathbf{F}_{v}=\left[\begin{array}{c}
0 \\
\tau_{x x} \\
\tau_{x y} \\
u \tau_{x x}+v \tau_{x y}-q_{x}
\end{array}\right], \mathbf{G}_{v}=\left[\begin{array}{c} 
\\
0 \\
\tau_{y x} \\
\tau_{y y} \\
u \tau_{y x}+v \tau_{y y}-q_{y}
\end{array}\right] .
\end{gathered}
$$

$\rho$ and $\mathrm{p}$ denote the density and pressure of the gas, respectively. $u, v$ denote the velocity components in the $\mathrm{x}, \mathrm{y}$ directions, respectively. The total energy of the unit volume is denoted as $E=\frac{p}{\gamma-1}$ $+\frac{1}{2} \rho\left(u^{2}+v^{2}\right)$. The state equation of an ideal gas is used: $p=\rho R T$, where $R=R_{0} / M_{\text {air }}, R_{0}=8.314 \mathrm{~J} /(\mathrm{mol} \mathrm{K})$, and $M_{\text {air }}$ denotes the molar mass of the gas.

The viscous stress components are as follows:

$$
\tau_{x x}=\mu\left(\frac{4}{3} \frac{\partial u}{\partial x}-\frac{2}{3} \frac{\partial v}{\partial y}\right), \quad \tau_{y y}=\mu\left(\frac{4}{3} \frac{\partial v}{\partial y}-\frac{2}{3} \frac{\partial u}{\partial x}\right) .
$$

The compositions of heat flow are as follows:

$$
q_{x}=-\frac{\mu}{\operatorname{Pr}(\gamma-1) M_{\infty}^{2}} \frac{\partial T}{\partial x}, \quad q_{y}=-\frac{\mu}{\operatorname{Pr}(\gamma-1) M_{\infty}^{2}} \frac{\partial T}{\partial y} .
$$


TABLE I. Boundary condition.

\begin{tabular}{lcccccc}
\hline \hline Boundary & $\mathrm{AB}$ & $\mathrm{EF}$ & $\mathrm{BC} / \mathrm{CD} / \mathrm{DE}$ & $\mathrm{HG}$ & $\mathrm{AH}$ & $\mathrm{FG}$ \\
\hline Condition & Incoming flow & Jet & Wall & Extrapolated & No-reflection & Symmetry \\
\hline \hline
\end{tabular}

TABLE II. Parameters of supersonic incoming flow.

\begin{tabular}{lccccc}
\hline \hline Component & $\mathrm{Ma}$ & Temperature (K) & Static pressure (Pa) & Density $\left(\mathrm{kg} \mathrm{m}^{-3}\right)$ & $\operatorname{Re}$ \\
\hline Air & 3.0 & 216.65 & $5.529 \times 10^{3}$ & $8.9 \times 10^{-2}$ & $5.542 \times 10^{6}$ \\
\hline \hline
\end{tabular}

After a Jacobian transformation, the two-dimensional Navier-Stokes equation in the curvilinear coordinate system is

$$
\frac{\partial \tilde{\boldsymbol{U}}}{\partial t}+\frac{\partial \tilde{\boldsymbol{F}}}{\partial \xi}+\frac{\partial \tilde{\boldsymbol{G}}}{\partial \eta}=\frac{1}{\operatorname{Re}}\left(\frac{\partial \tilde{\boldsymbol{F}}_{v}}{\partial \xi}+\frac{\partial \tilde{\boldsymbol{G}}_{v}}{\partial \eta}\right) .
$$

The vector forms are as follows:

$$
\begin{aligned}
& \tilde{\boldsymbol{U}}=\frac{1}{J} \mathbf{U}, \tilde{\boldsymbol{F}}=\frac{1}{J}\left(\xi_{x} \mathbf{F}+\xi_{y} \mathbf{G}\right), \tilde{\boldsymbol{G}}=\frac{1}{J}\left(\eta_{x} \mathbf{F}+\eta_{y} \mathbf{G}\right), \\
& \tilde{\boldsymbol{F}}_{v}=\frac{1}{J}\left(\xi_{x} \mathbf{F}_{v}+\xi_{y} \mathbf{G}_{v}\right), \tilde{\boldsymbol{G}}_{v}=\frac{1}{J}\left(\eta_{x} \mathbf{F}_{v}+\eta_{y} \mathbf{G}_{v}\right) .
\end{aligned}
$$

The Jacobian determinant is

$$
J=\left|\frac{\partial(\xi, \eta)}{\partial(x, y)}\right|=\frac{1}{x_{\xi} y_{\eta}-x_{\eta} y_{\xi}} .
$$

\section{Numerical method}

The discrete scheme of the convective term is

$$
\mathbf{C O N V}_{i, j}^{n}=\frac{\left(\tilde{\boldsymbol{F}}_{i+\frac{1}{2}, j}^{n}-\tilde{\boldsymbol{F}}_{i-\frac{1}{2}, j}^{n}\right)}{\Delta x}+\frac{\left(\tilde{\boldsymbol{G}}_{i, j+\frac{1}{2}}^{n}-\tilde{\boldsymbol{G}}_{i, j-\frac{1}{2}}^{n}\right)}{\Delta y} .
$$

The flux vectors are as follows:

$$
\left\{\begin{array}{l}
\tilde{\boldsymbol{F}}_{i+\frac{1}{2}, j}=\tilde{\boldsymbol{F}}_{i+\frac{1}{2}, j}^{+}+\tilde{\boldsymbol{F}}_{i+\frac{1}{2}, j}^{-} \\
\tilde{\boldsymbol{G}}_{i, j+\frac{1}{2}}=\tilde{\boldsymbol{G}}_{i, j+\frac{1}{2}}^{+}+\tilde{\boldsymbol{G}}_{i, j+\frac{1}{2}}^{-}
\end{array}\right.
$$

The $\tilde{\boldsymbol{F}}_{i+\frac{1}{2}, j}^{ \pm}$and $\tilde{\boldsymbol{G}}_{i+\frac{1}{2}, j}^{ \pm}$were configured by the fifth order WENO scheme

$$
\begin{aligned}
& \tilde{\boldsymbol{F}}_{i+\frac{1}{2}, j}^{ \pm}=\sum_{k=0}^{2} \omega_{x k}^{ \pm} q_{x k}^{ \pm}, \\
& \tilde{\boldsymbol{G}}_{i+\frac{1}{2}, j}^{ \pm}=\sum_{k=0}^{2} \omega_{y k}^{ \pm} q_{y k}^{ \pm} .
\end{aligned}
$$

The weighting factor suggested by Jiang and $S h \mathrm{u}^{15}$ was used in this paper. The method of Jiang and Shu is given in the Appendix.

The viscous terms are as follows:

$$
\operatorname{VISC}_{i, j}^{n}=\frac{\partial \tilde{\boldsymbol{F}} v_{i, j}^{n}}{\partial x}+\frac{\partial \tilde{\boldsymbol{G}} v_{i, j}^{n}}{\partial y} .
$$

The derivative terms can be discretely expressed by the sixth order central difference scheme as follows:

$$
\frac{\partial f_{i}}{\partial x}=\frac{1}{60 \Delta x}\left[f_{i+3}-f_{i-3}-9\left(f_{i+2}-f_{i-2}\right)+45\left(f_{i+1}-f_{i-1}\right)\right] .
$$

In this study, the third-order precision Runge-Kutta method was used to calculate the time integral and the Courant-Friedrichs-Lewy (CFL) number is 0.1 ,

$$
\left\{\begin{array}{l}
y_{n+1}=y_{n}+h\left(\lambda_{1} K_{1}+\lambda_{2} K_{2}+\lambda_{3} K_{3}\right), \\
K_{1}=f\left(x_{n}, y_{n}\right), \\
K_{2}=f\left(x_{n}+p h, y_{n}+p h K_{1}\right), \\
K_{3}=f\left(x_{n}+q h, y_{n}+q h\left(r K_{1}+s K_{2}\right)\right) .
\end{array}\right.
$$

TABLE III. Parameters of the jet.

\begin{tabular}{lcccccc}
\hline \hline No. & $\begin{array}{c}\text { Temperature } \\
(\mathrm{K})\end{array}$ & $\begin{array}{c}\text { Static pressure ratio } \\
\left(p_{\text {jet }} / p_{\infty}\right)\end{array}$ & $\begin{array}{c}\text { Static pressure } \\
(\mathrm{Pa})\end{array}$ & $\begin{array}{c}\text { Density } \\
\left(\mathrm{kg} \mathrm{m}^{-3}\right)\end{array}$ & $\mathrm{Ma}$ & $\mathrm{Re}_{\text {jet }}$ \\
\hline 1 & 1000 & 0.6 & $3.314 \times 10^{3}$ & $1.16 \times 10^{-2}$ & 2.0 & $1.034 \times 10^{6}$ \\
2 & 1000 & 1.2 & $6.635 \times 10^{3}$ & $2.314 \times 10^{-2}$ & 2.0 & $2.063 \times 10^{6}$ \\
3 & 1000 & 2.5 & $1.382 \times 10^{4}$ & $4.82 \times 10^{-2}$ & 2.0 & $4.297 \times 10^{6}$ \\
4 & 1000 & 10 & $5.529 \times 10^{4}$ & 0.1928 & 2.0 & $1.719 \times 10^{7}$ \\
5 & 1000 & 30 & $1.659 \times 10^{5}$ & 0.3216 & 2.0 & $2.867 \times 10^{7}$ \\
\hline \hline
\end{tabular}




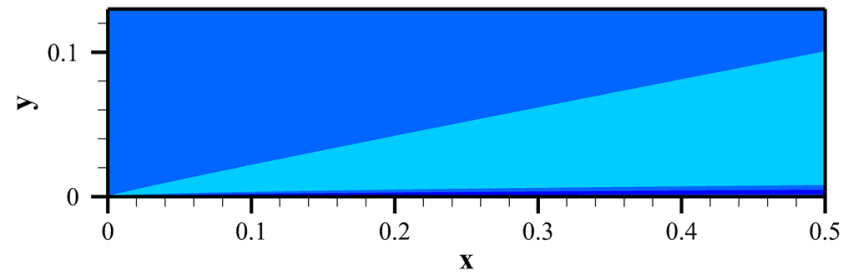

FIG. 2. Density of the supersonic flow on the plate.

\section{Algorithm verification and verification of grid independence}

The case of supersonic flow on the plate was used to verify the correctness of the calculation method. The supersonic flow Mach number is 7, and the Reynolds number is $1.233 \times 10^{6}$. As shown in Fig. 2, the density of the supersonic flow becomes smaller after the front edge shock and the boundary layer gradually thickens along the direction of the supersonic incoming flow. This is consistent with the actual situation of supersonic flow on the plate.

The self-similar solution for the compressible boundary layer was used for comparison. The self-similar solution for the compressible boundary layer is a semianalytical solution. In this paper, a fifth order Runge-Kutta method combined with a shooting method was adopted to solve the self-similar solution. Hence, the comparisons between the semianalytical solution and numerical solution were carried out to validate the code and algebraic method of the numerical simulations. As shown in Fig. 3, the curve of the numerical simulation agrees well with the curve of the semianalytical solution. This shows that the algorithm is reliable.

Grid independence tests were conducted to determine the suitable computational mesh for the supersonic flow and jet interaction problems. The static pressure ratio $p_{\text {jet }} / p_{\infty}=2.5$, and the aspect ratio $\mathrm{DE} / \mathrm{CD}=2: 1$. Data on $\mathrm{y}=0$ are chosen to verify grid-independence.

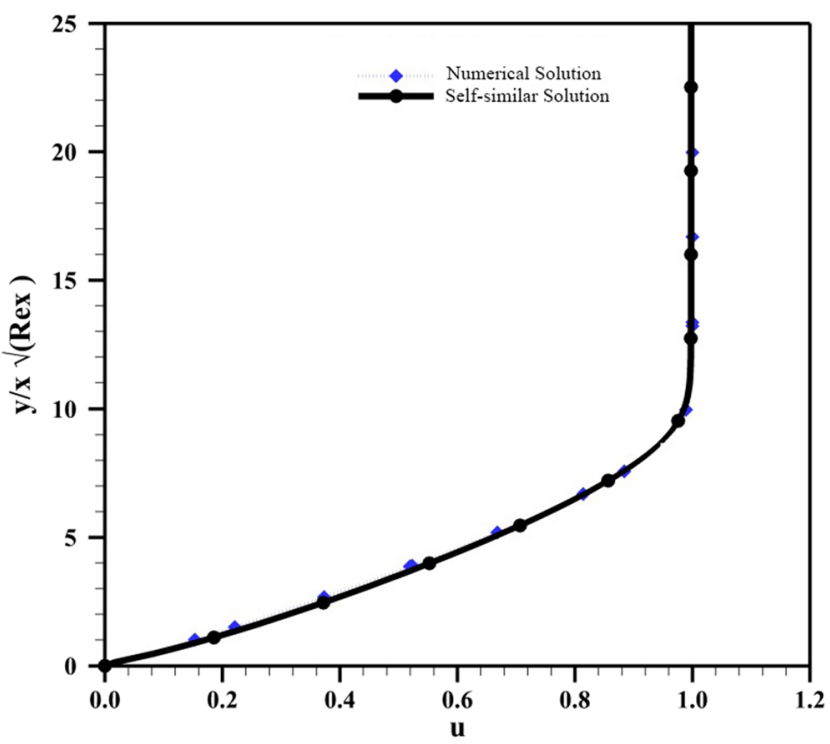

FIG. 3. Velocity profile in the boundary layer.

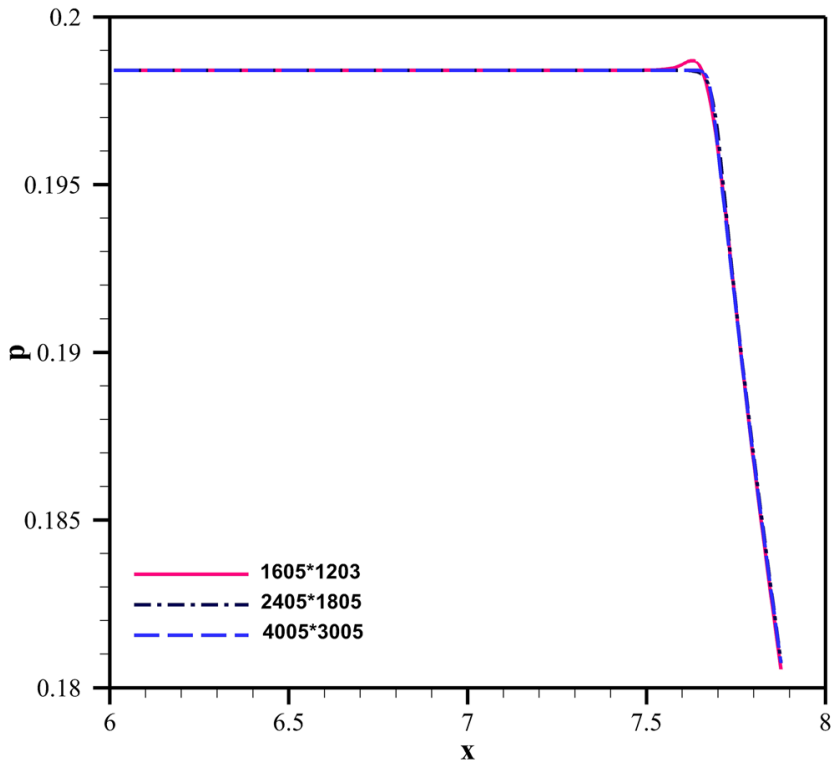

FIG. 4. Static pressure profile of the jet for different grids $(y=0, D E / C D=2: 1$, $p_{\text {jet }} / p_{\infty}=2.5$, and $\mathrm{t}=0.015 \mathrm{~s}$ ).

The static pressure profile and temperature profile of the symmetrical line position are determined for $\mathrm{t}=0.015 \mathrm{~s}$. Three different grids are used $(1605 \times 1203,2405 \times 1805$, and $4005 \times 3005)$.

Figures 4 and 5 show the pressure profile and temperature profile, respectively, for the different grids. The results of the 3 sets of grids are in good agreement. Thus, the grid independence has been verified and we choose $1605 \times 1203$ grids for the following numerical simulation.

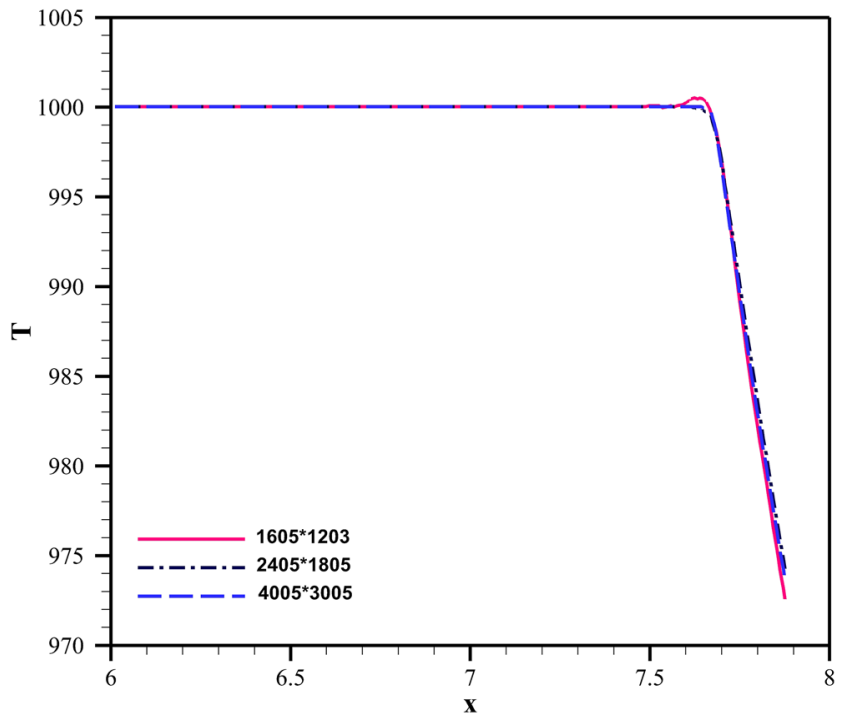

FIG. 5. Temperature profile of the jet for different grids $\left(y=0, D E / C D=2: 1, p_{\text {jet }} / p_{\infty}\right.$ $=2.5$, and $\mathrm{t}=0.015 \mathrm{~s}$ ). 


\section{RESULTS AND DISCUSSION}

\section{A. Basic flow field structure}

The double backward-facing steps are used to simulate the interaction of the two shear layers. The DE is twice as long as CD as shown in Fig. 6. We use the length of $\mathrm{CD}$ as the dimensionless unit of length.

Above the first backward-facing step at $\mathrm{BC}$, the supersonic incoming flow occurs with $\mathrm{Ma}=3$. The supersonic incoming flow is separated from the vertical plane of the first back step. The extension of the wall boundary layer in the direction of the flow forms a shear layer over the first backward-facing step, which is named the supersonic incoming flow shear layer.

A high-temperature and high-pressure jet with $\mathrm{Ma}=2$ occurs on the right side of the second backward-facing step at EF. The jet passes through the triangle-shaped constant velocity core zone, where the temperature, density, etc., are constant. The jet is separated at the corner of the steps and rapid expansion occurs near the steps at the corner. Because the outlet static pressure and the ambient static pressure do not match, an expansion sector is formed at the beginning of the jet. The streamline gradually changes direction in the expansion sector until the fluid exits the expansion sector. A shear layer, called a jet shear layer, is formed outside the expansion sector.

A mixing zone is formed between the jet shear layer and the supersonic incoming flow shear layer. The flow velocity of the airflow in this area is small and the kinetic energy is converted into internal energy. As a result of the complex interactions between the jet shear layer and the supersonic incoming flow shear layer, complex wave structures such as vortices and oscillation shock waves are formed as shown in Fig. 7. The temperature is higher in the mixing zone than in the other regions.

Due to the interaction between the jet shear layer and the supersonic incoming shear layer, a jet impinges on the recirculation zone between the two shear layers; the jet is named as the secondary jet. The secondary jet is ejected periodically; this phenomenon is

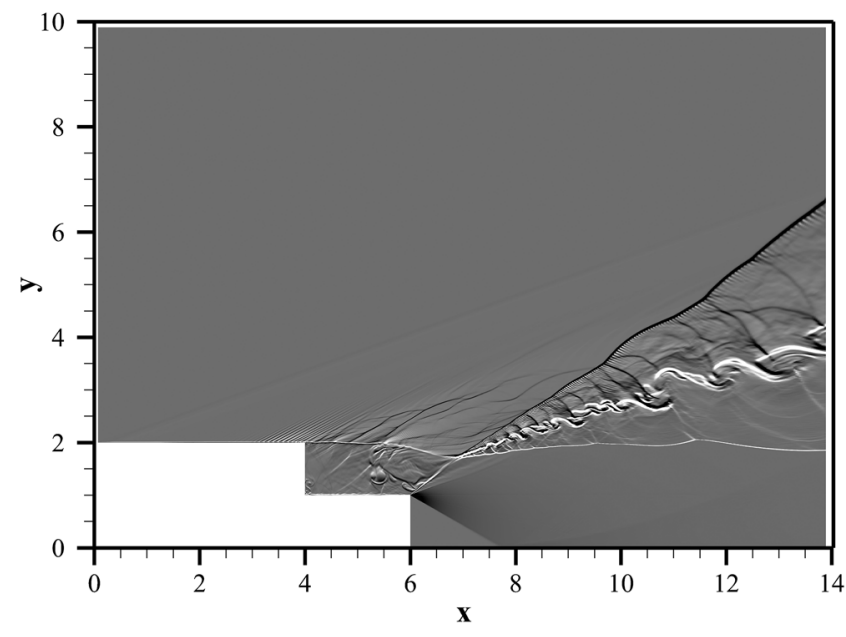

FIG. 6. Density gradient of the fundamental phenomenon $\left(D E / C D=2: 1, p_{\text {jet }} / p_{\infty}\right.$ $=2.5$ ).

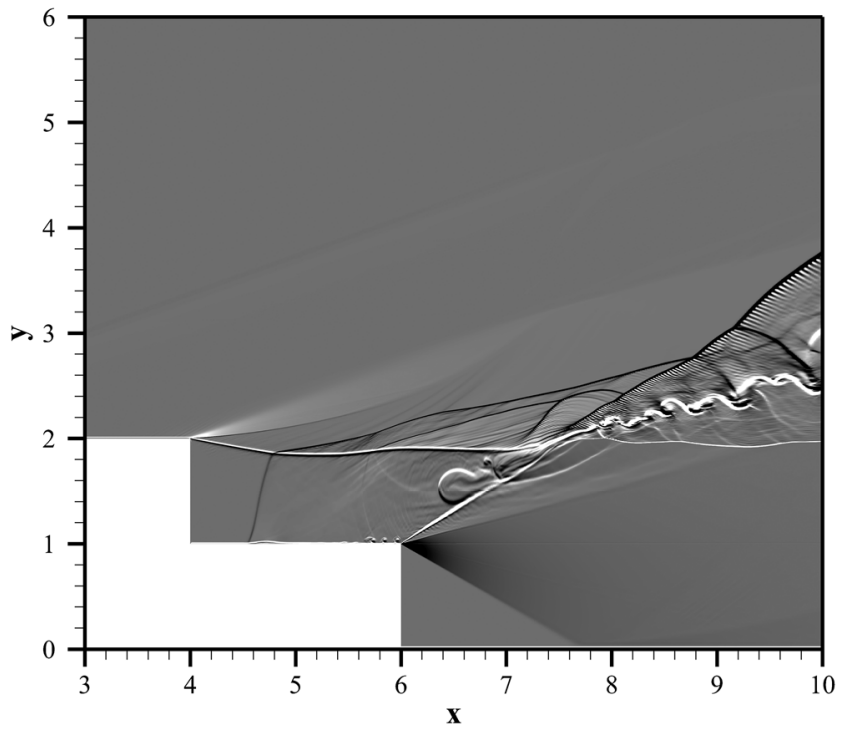

FIG. 7. The secondary jet flow and the secondary shock wave $(D E / C D=2: 1$, $\left.p_{\text {jet }} / p_{\infty}=2.5\right)$.

explained in Secs. III B-III D. The periodicity of the secondary jet is related to the fluctuation of the shear layers. When the horizontal inclination of the jet shear layer reaches a certain level, a new secondary jet is ejected.

The secondary jet is generated by the interaction between the supersonic incoming flow shear layer and the jet shear layer; a weak shock wave, called a secondary shock wave, is produced in front of the secondary jet as shown in Fig. 7. The secondary shock wave oscillates in the mixing zone as shown in Fig. 8. The secondary shock wave moves to the right and reflects off the wall CD and then moves to the left to react with substances that have been injected. Hence the secondary shock wave has a certain effect on the diffusion of the secondary jet in the mixing zone. In other words, the secondary jet produces the secondary shock wave, which, in turn, affects the secondary jet. Due to the periodicity of the secondary jet, the secondary shock wave also occurs periodically. This means that the secondary shock wave oscillates regularly in the mixing zone.

The movement of the secondary shock waves also affects the morphology of the supersonic incoming flow shear layer. If the secondary shock wave moves to the right, the incoming flow shear layer in front of the secondary shock wave will be raised. On the other hand, if the secondary shock wave moves to the left, the incoming flow shear layer in front of the secondary shock wave will be lowered. On the other side of the incoming flow shear layer, the secondary shock wave also produces its own transmit shock.

There are shocklets in the boundary layer as shown in Fig. 8(a). As the supersonic incoming flow shear layer passes over the backward-facing step, a mixing zone is formed, and the flow in the mixing zone is subsonic. Disturbance is transmitted from the place below the speed of sound to the boundary layer on the step. Shocklets are generated by these disturbances.

After the interaction between the two shear layers, they converge into a shear layer called a mixed shear layer. The mixed shear 


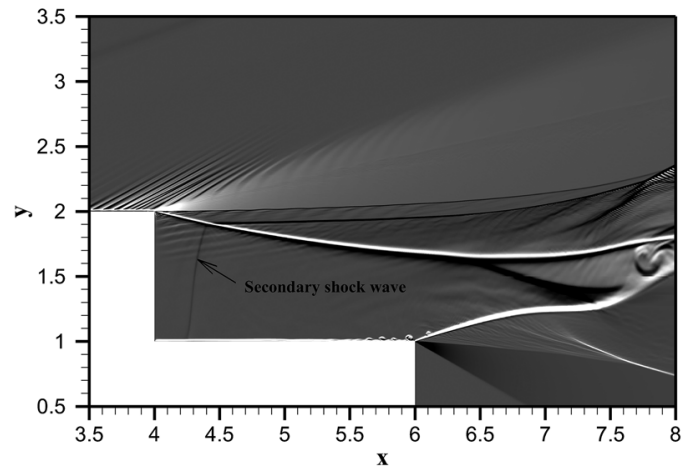

(a)

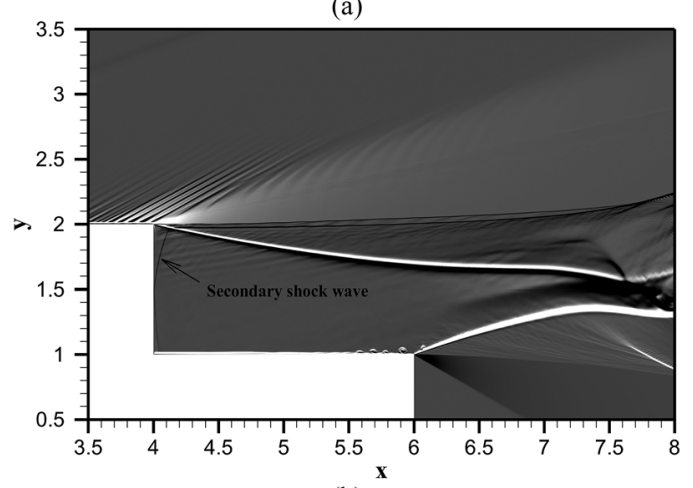

(b)

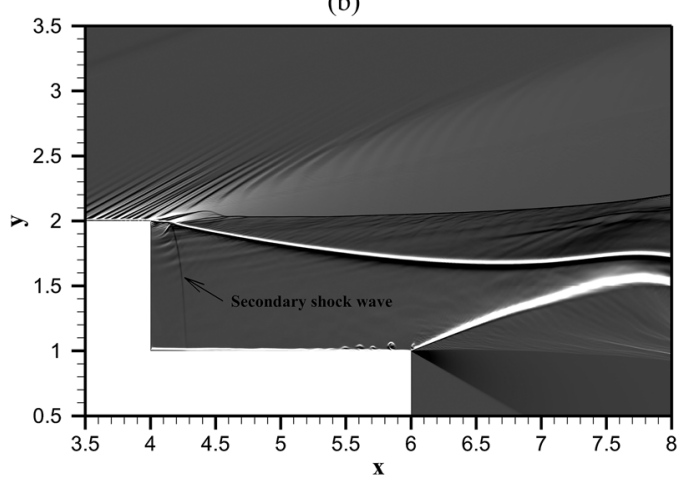

(c)

FIG. 8. The process of secondary shock wave reflection on the wall $(D E / C D$ $\left.=2: 1, p_{\text {iet }} / p_{\infty}=2.5\right)$ : (a) the secondary shock wave moving toward the wall $(t=0.016 \mathrm{~s}),(b)$ interaction between the secondary shock wave and the wall interaction $(t=0.017 \mathrm{~s})$, and $(c)$ the secondary shock wave moving away from the wall $(\mathrm{t}=0.018 \mathrm{~s})$.

layer is broken because of instability, and irregular and unpaired vortex structures are formed as shown in Fig. 9. The vortices are formed in the mixed shear layer, which results in the fragmentation of the mixed shear layer. Two shock waves are formed on both sides of the mixed shear layer. Because the static pressure and density of the vortices are lower than those of the surrounding environment, a pressure difference is created. The fluctuation in the pressure causes a disturbance to the shear layer and shocklets appear on both sides of the mixed shear layer. Strong compression waves are formed on both sides of the vortex structure, and the compression effect

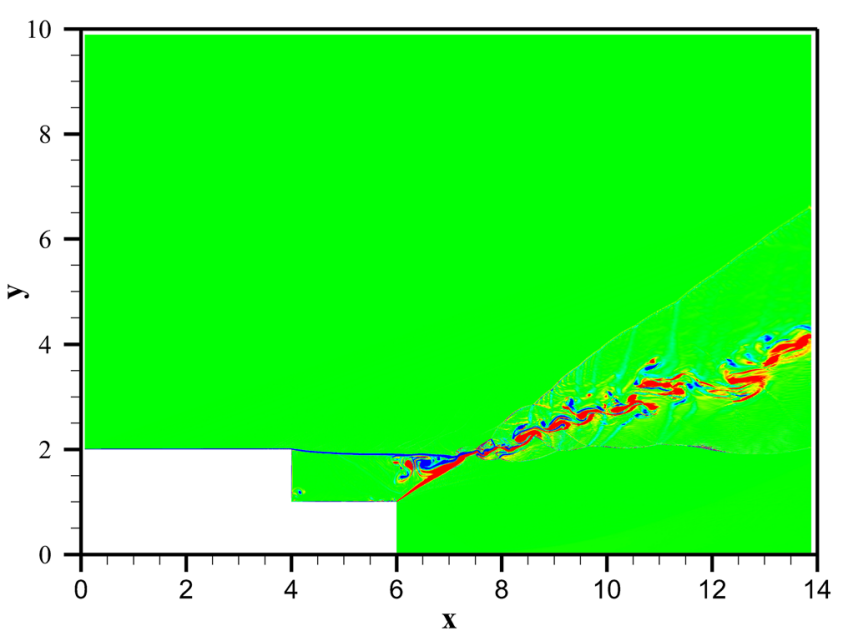

FIG. 9. Vortices created by the interaction of the layers $\left(D E / C D=2: 1, p_{\text {jet }} / p_{\infty}\right.$ $=2.5$ ).

causes the compression wave system to gradually aggregate to form shocklets.

At the same time, due to the effect of the secondary shock wave and the lower wall boundary layer as shown in Fig. 10, there are initial disturbances at the interface of the two different fluids. After the shock waves accelerate, the perturbations at the interface will increase over time. The secondary shock wave provides initial velocity to the jet shear layer, and the secondary shock wave begins to move in the direction of the secondary shock wave. The interfacial perturbations increase and gradually develop into a classical

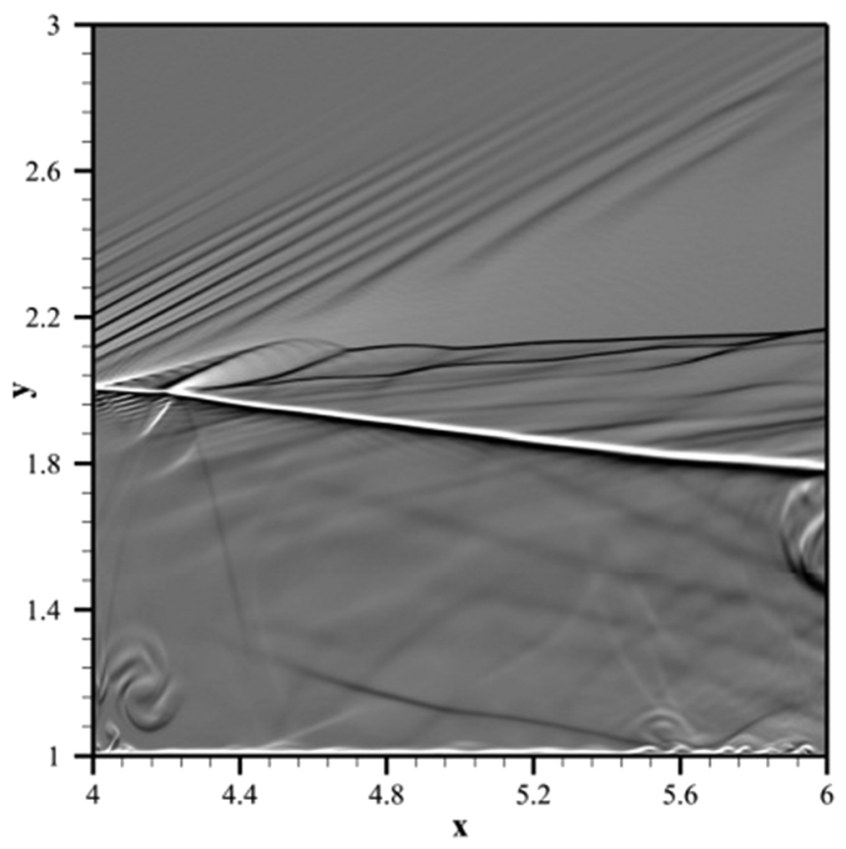

FIG. 10. Mushroom structure in the mixing zone $\left(D E / C D=2: 1, p_{\text {jet }} / p_{\infty}=2.5\right)$. 


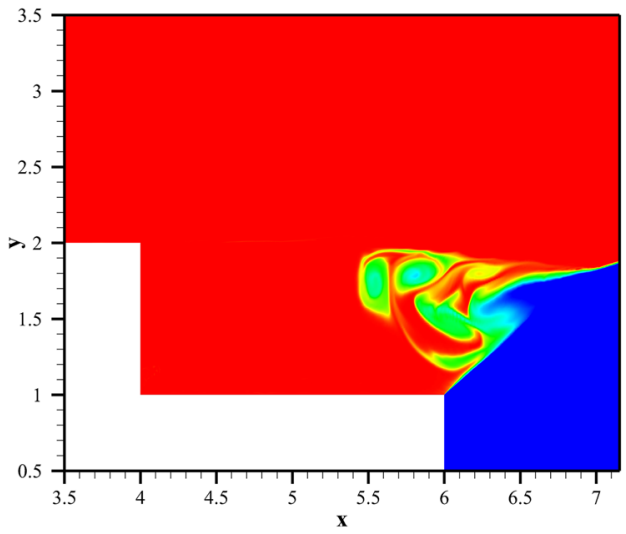

(a)

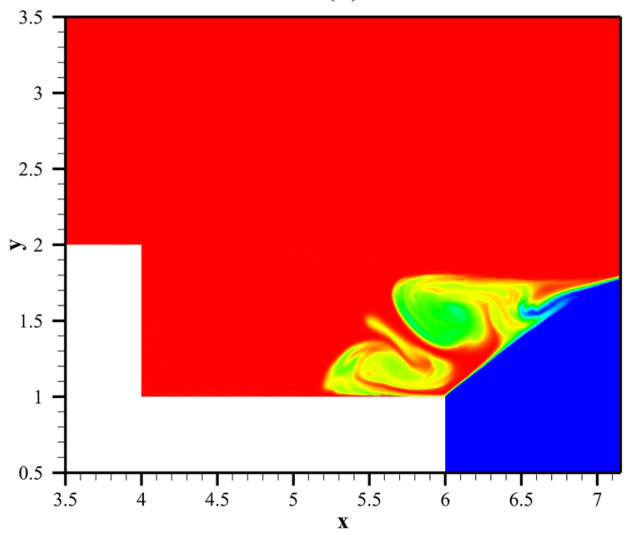

(c)

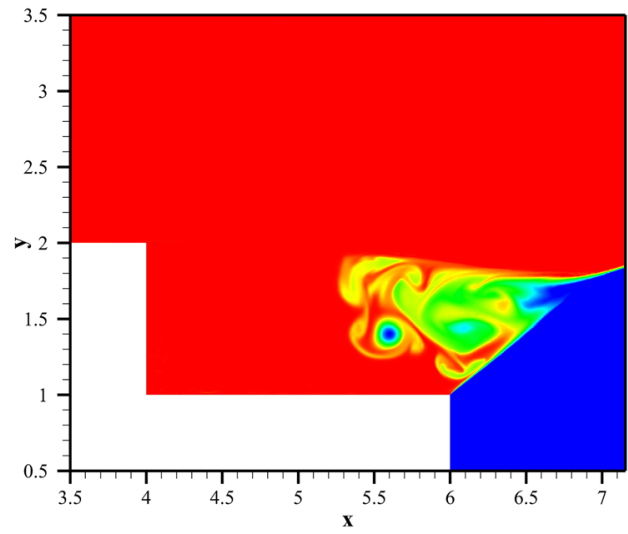

(b)

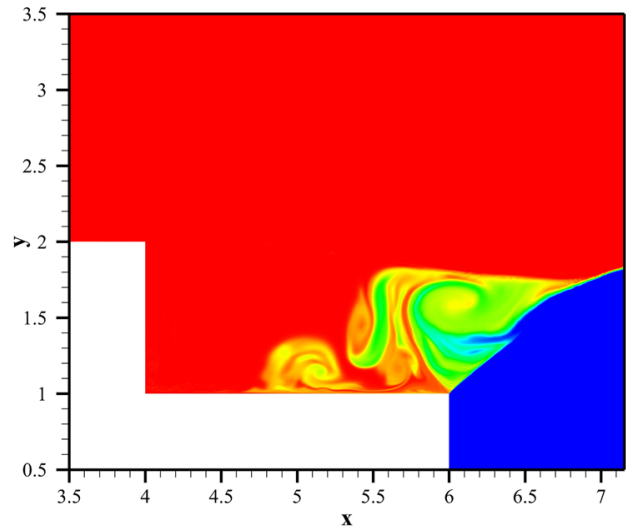

(d)
FIG. 11. The secondary jet emerges at (a) $0.016 \mathrm{~s}$, (b) $0.042 \mathrm{~s}$, (c) $0.066 \mathrm{~s}$, and (d) $0.089 \mathrm{~s}\left(\mathrm{DE} / \mathrm{CD}=2: 1, p_{\mathrm{jet}} / p_{\infty}=10\right)$. mushroom structure under the action of the shock-induced pressure gradient. The mushroom structures are observed at the junction of the walls CD and DE. These mushroom structures are components of the jet shear layer that are accelerated by the secondary shock wave.

\section{B. Fluctuations of the shear layers}

Owing to the relationship between the periodicity of the secondary shock wave and the fluctuations of the shear layers, it is necessary to determine the fluctuations of the shear layers first. The angle between the jet shear layer and the x-axis is defined as a horizontal angle.

The shear layer oscillation is investigated at a static pressure ratio of 10 . At $t=0.036 \mathrm{~s}$, the minimum horizontal angle is $29^{\circ}$, and at $\mathrm{t}=0.045 \mathrm{~s}$, the maximum horizontal angle is $48^{\circ}$. The component calibration method is used to observe the secondary jet. The horizontal angle of the jet shear layer periodically increases and decreases, causing the secondary jet to emerge periodically. In the first cycle, when the horizontal angle is greater than $40^{\circ}$, the secondary jet emerges. When the horizontal angle decreases, the secondary jet begins to weaken until it stops.

During $0.1 \mathrm{~s}$, the secondary jet emerges 4 times, i.e., at $0.016 \mathrm{~s}$, $0.042 \mathrm{~s}, 0.066 \mathrm{~s}$, and $0.089 \mathrm{~s}$. As shown in Fig. 11, the phenomenon that the secondary jet enters the mixing zone at these four moments can be clearly observed. The component calibration method is used to observe the secondary jet. Because we consider the jet and supersonic flow of air, we can use the component calibration method. Since the components of the jet and supersonic flow are the same. We define $s 1=\rho 1 /(\rho 1+\rho 2)$ and $s 2=\rho 2 /(\rho 1+\rho 2)$. If $s 1=1$ and $s 2=0$, this means that the flow is the jet. If $s 1=0$ and $s 2=1$, the flow is the supersonic incoming flow completely. If $s 1=0$ and $s 2=1$, the flow is the supersonic incoming flow completely. If $0<\mathrm{s} 1<1$ and $0<\mathrm{s} 2<1$, it means that mixing has occurred in the flow. This method can well observe the generation and evolution of secondary

TABLE IV. Horizontal angles of the jet shear layer when the secondary jet is produced.

\begin{tabular}{lcccc}
\hline \hline$p_{\text {jet }} / p_{\infty}$ Period & & & & \\
1.2 & $1(\mathrm{deg})$ & $2(\mathrm{deg})$ & $3(\mathrm{deg})$ & $4(\mathrm{deg})$ \\
2.5 & 33.9 & 35.6 & 34.2 & 34.4 \\
10 & 40.8 & 41.0 & 38.2 & 39.0 \\
30 & 43.9 & 41.0 & 38.5 & 39.2 \\
\hline \hline
\end{tabular}




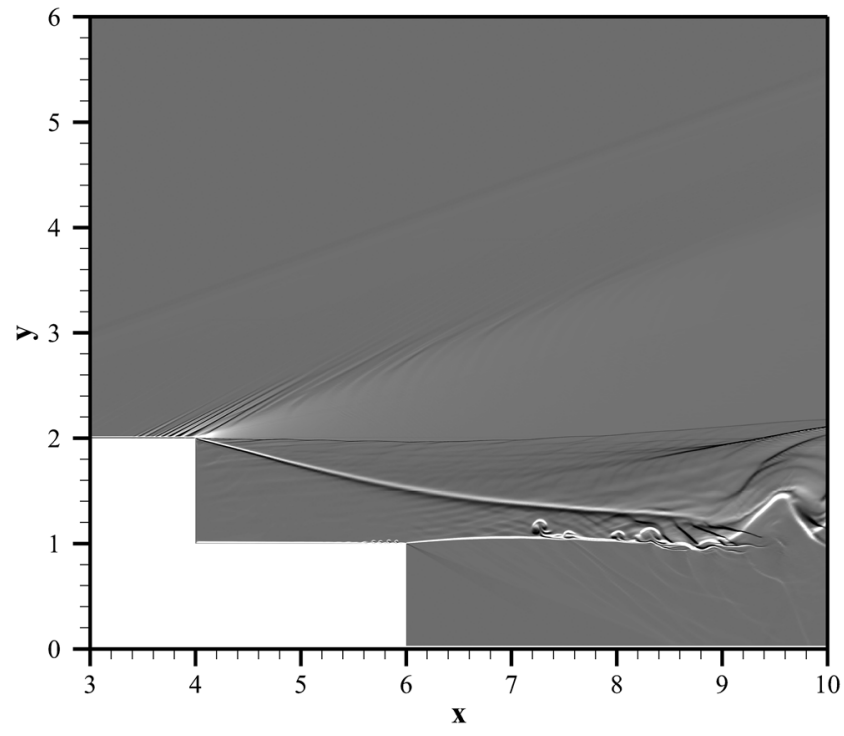

FIG. 12. Density gradient of the fundamental phenomenon $\left(D E / C D=2: 1, p_{\text {jet }} / p_{\infty}\right.$ $=0.6$ ).

jets, and hence, it is used to visualize the injection process of the secondary jets.

As shown in Table IV, the horizontal angles of the jet shear at a static pressure ratio of 2.5 are $40.8^{\circ}, 41.0^{\circ}, 38.2^{\circ}$, and $39.0^{\circ}$

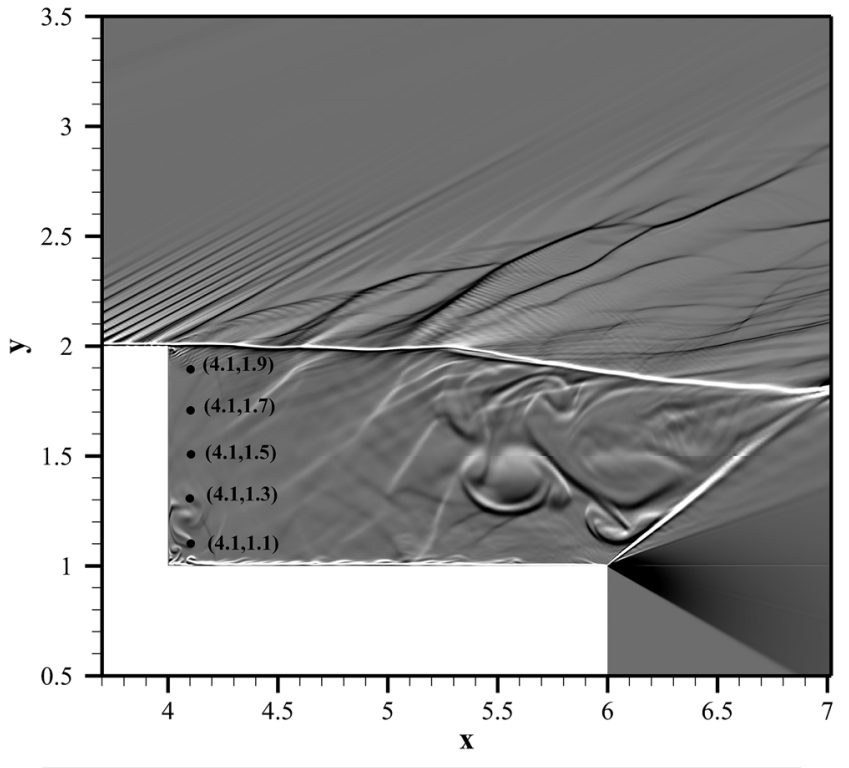

FIG. 14. Five pressure points near the wall ( $\left.D E / C D=2: 1, p_{\mathrm{jet}} / p_{\infty}=2.5\right)$.

at $0.016 \mathrm{~s}, 0.042 \mathrm{~s}, 0.066 \mathrm{~s}$, and $0.089 \mathrm{~s}$. This demonstrates that the secondary jet emerges when the horizontal angle is greater than a certain value, and this is similar for both static pressure ratios.

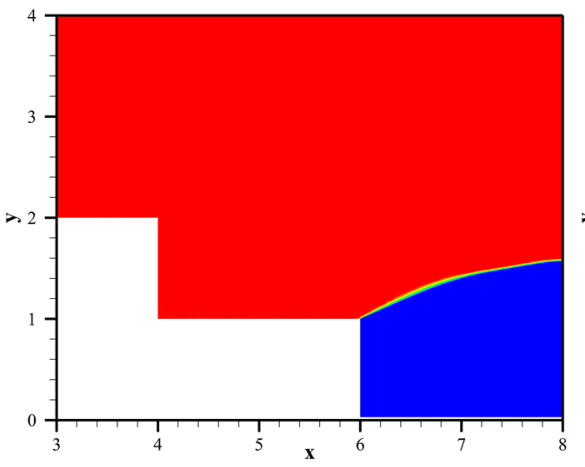

(a)

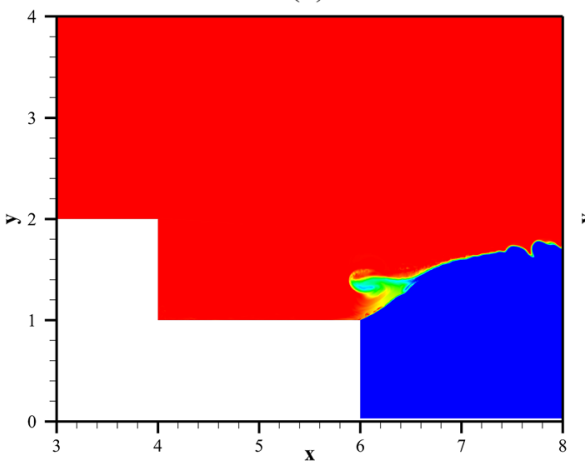

(d)

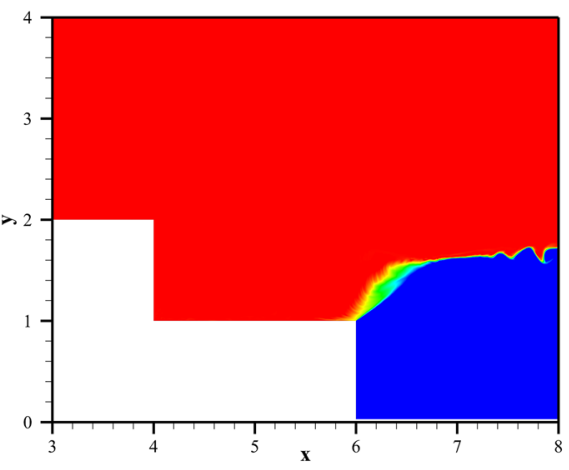

(b)

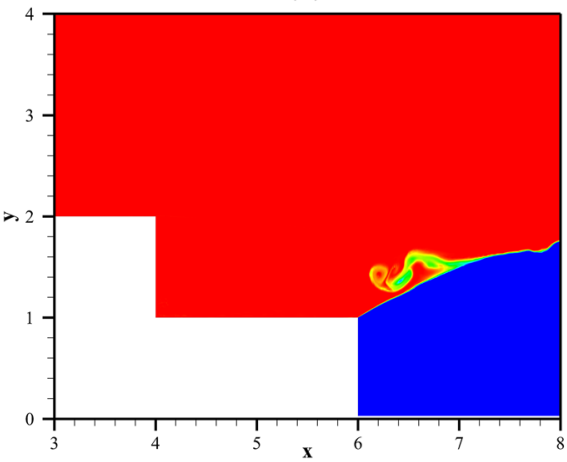

(e)

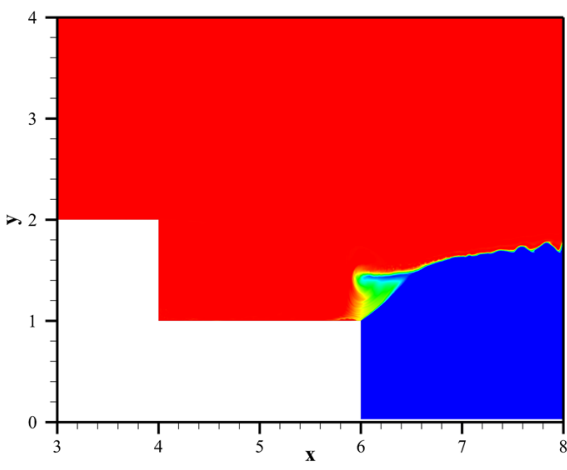

(c)

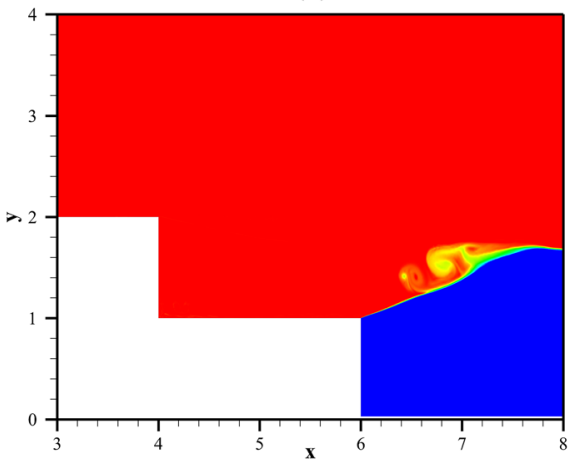

(f)

FIG. 13. Generation and disappearance of the secondary jet: (a) $0.024 \mathrm{~s}$, (b) $0.026 \mathrm{~s}$, (c) $0.027 \mathrm{~s}$, (d) $0.028 \mathrm{~s}$, (e) $0.030 \mathrm{~s}$, and (f) $0.036 \mathrm{~s}\left(\mathrm{DE} / \mathrm{CD}=2: 1, p_{\mathrm{jet}} / p_{\infty}=2.5\right)$. 


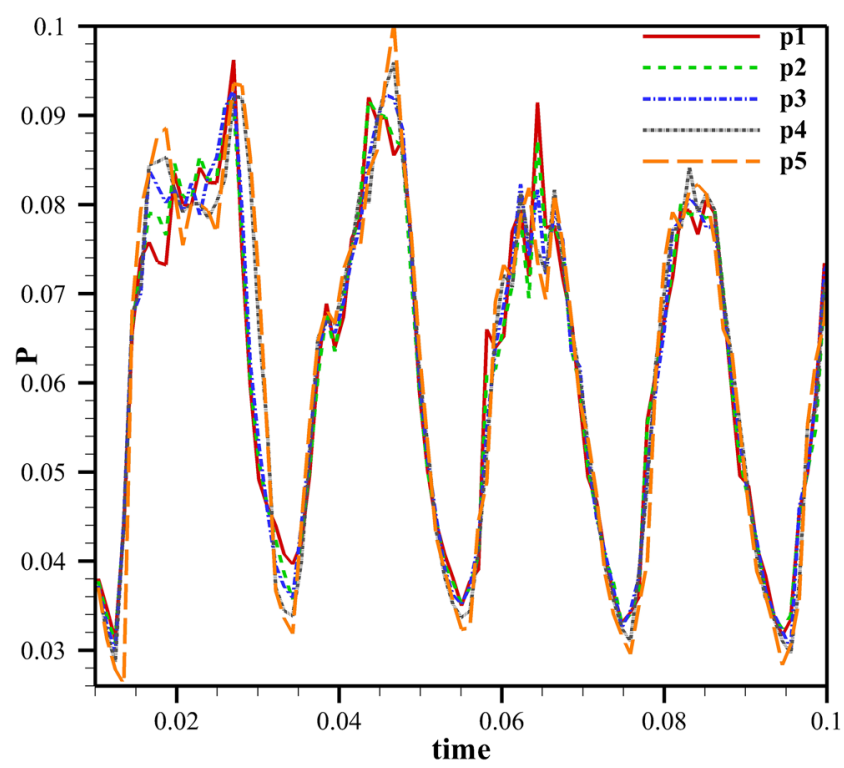

FIG. 15. The distribution of the five pressure points over time $\left(D E / C D=2: 1, p_{\text {jet }} / p_{\infty}\right.$ $=2.5$ ).
If the pressure is relatively small, especially $p_{\text {jet }} / p_{\infty}=2.5$ and 10 , the horizontal shear angle when the secondary jet is produced grows slow and is almost the same. However, overall, the horizontal shear angle when secondary jet is produced increases as the static pressure ratio increases as shown in Table IV. The horizontal angles of the jet shear at a static pressure ratio of 10 are $55.7^{\circ}, 54.2^{\circ}, 54.2^{\circ}$, and $54.1^{\circ}$; these horizontal angles are obviously larger than the horizontal angles at other static pressure ratios.

\section{Over-expanded jet}

A shear layer oscillating at a static pressure ratio of 0.6 was investigated. In this case, the pressure of the jet is lower than the pressure of the surroundings, i.e., the jet is an over-expanded jet. The jet and supersonic flow interact at the second step to form a complex flow field structure. At $0.1 \mathrm{~s}$, even though the jet shear layer oscillates, there is no secondary jet between the jet shear layer and the supersonic incoming flow shear layer. This indicates that a secondary jet is not generated in the case of an over-expanded jet. Due to the periodical fluctuations of the shear layers, the shock waves oscillate in the mixing zone. In this condition, the jet shear layer oscillates irregularly and aperiodically. As Fig. 12 shows, because there are irregular fluctuations in the jet shear layer, shock waves

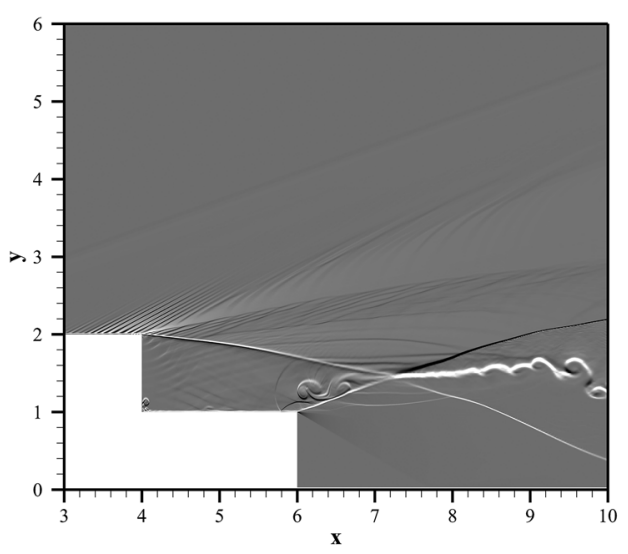

(a)

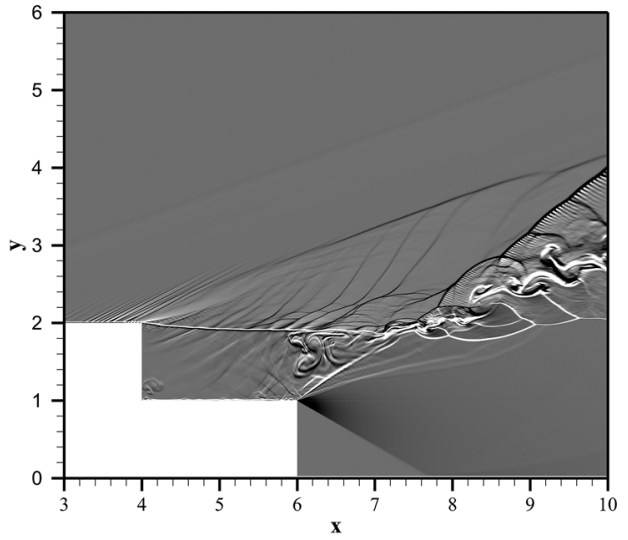

(c)

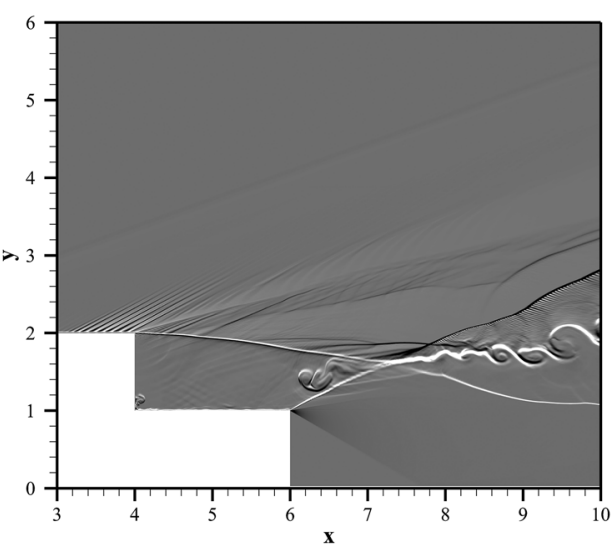

(b)

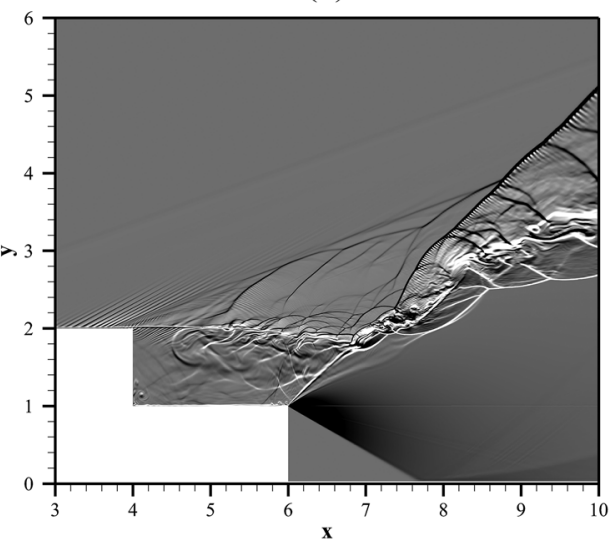

(d)
FIG. 16. Density gradients for different static pressure ratios: (a) $p_{\text {jet }} / p_{\infty}=1.2$, (b) $p_{\text {iet }} / p_{\infty}=2.5$, (c) $p_{\text {iet }} / p_{\infty}=10$, and (d) $p_{\text {jet }} / p_{\infty}=30(\mathrm{DE} / \mathrm{CD}=2: 1)$. 
generate in the mixing zone. These shock waves accelerate the components of the jet shear layer, and these components begin to move along the secondary shock wave's direction. The interfacial perturbations increase and gradually develop into a classical mushroom structure under the action of the shock-induced pressure gradient.

Mushroom structures are observed in the jet shear layer as shown in Fig. 12. They are the result of the jet shear layer that is accelerated by the shock waves due to the periodical fluctuation of the shear layers. The impact force, especially the shock wave that affects the shear layer, causes small perturbations. Over time, these small perturbations develop into flow mixing. As the disturbance increases, a nail-shaped structure is observed at the shear layer of the jet.

\section{Under-expanded jet}

The shear layer oscillations are investigated at static pressure ratios of 1.2, 2.5, 10, and 30. In these five cases, the pressure of the jet is higher than the pressure of the surroundings. This indicates that the jet is an under-expanded jet.

The example of the static pressure ratio of 2.5 is analyzed in this section. From $0.01 \mathrm{~s}$ to $0.1 \mathrm{~s}$, four periods are observed and each period lasts about $0.025 \mathrm{~s}$. The case of secondary jet injection is selected between $0.024 \mathrm{~s}$ and $0.036 \mathrm{~s}$.

The component calibration method is used to observe the secondary jet. Figure 13 shows the statuses of different times in the first period. The jet that is ejected from the left end of the second step is shown in red. The supersonic incoming flow above the first step is shown in blue. If the color between the jet shear layer and the supersonic flow shear layer is golden, the components of both layers have been mixed. This demonstrates the process of production of the secondary jet, which occurs at $0.027 \mathrm{~s}$. We can clearly see the gold components between the jet shear layer and the supersonic flow shear in the mixed zone. From $0.024 \mathrm{~s}$ to $0.026 \mathrm{~s}$, the components of the jet are rolling backward due to the fluctuation of the jet shear layer. Subsequently, from $0.026 \mathrm{~s}$ to $0.036 \mathrm{~s}$, as the jet shear layer rises upward, the new secondary jet appears at $0.027 \mathrm{~s}$. Between the jet shear layer and the supersonic flow shear layer, this new jet appears in blue. After the secondary jet occurs, the horizontal portion of the jet shear layer declines and the jet shear layer is depressed; the components that were previously ejected are rolled backward again. Subsequently, a new period begins and the process starts from the beginning.

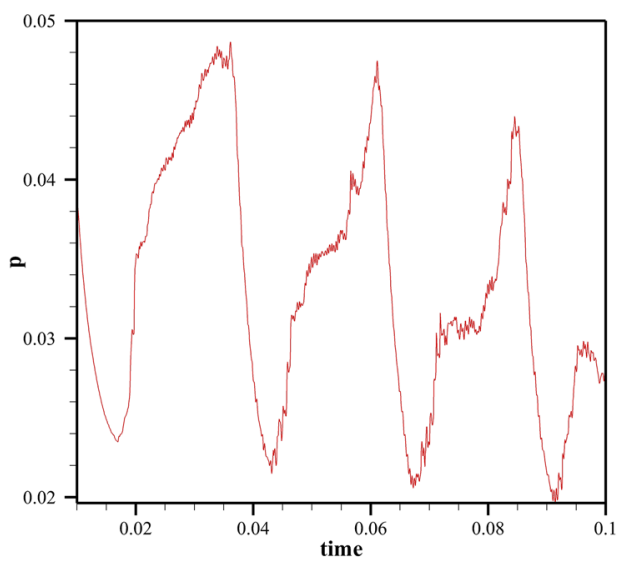

(a)

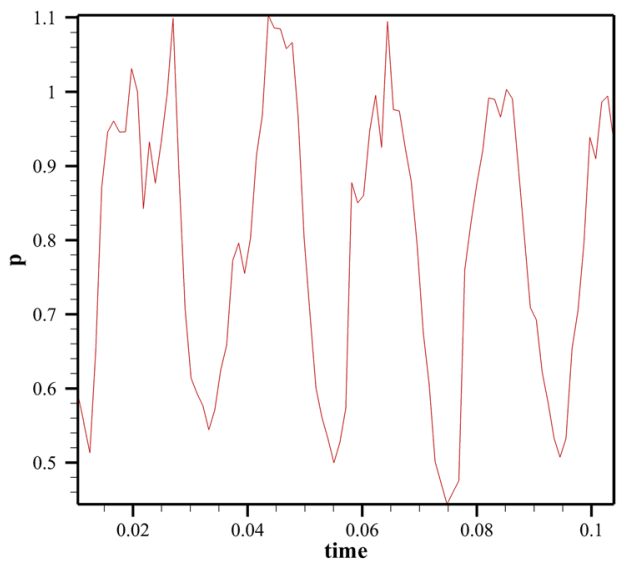

(c)

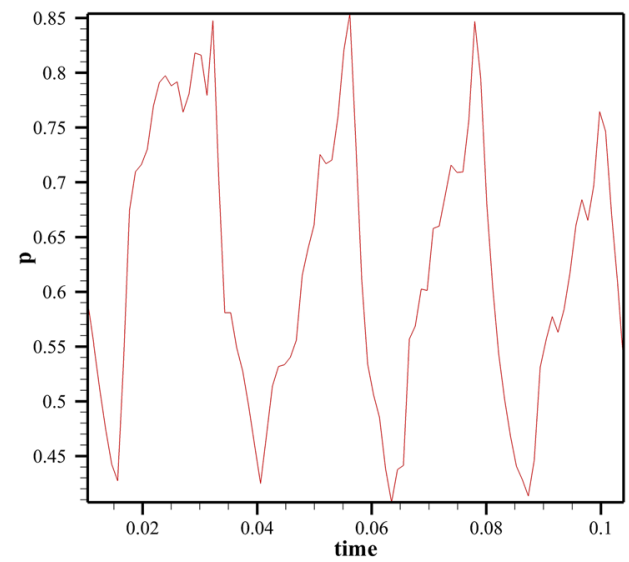

(b)

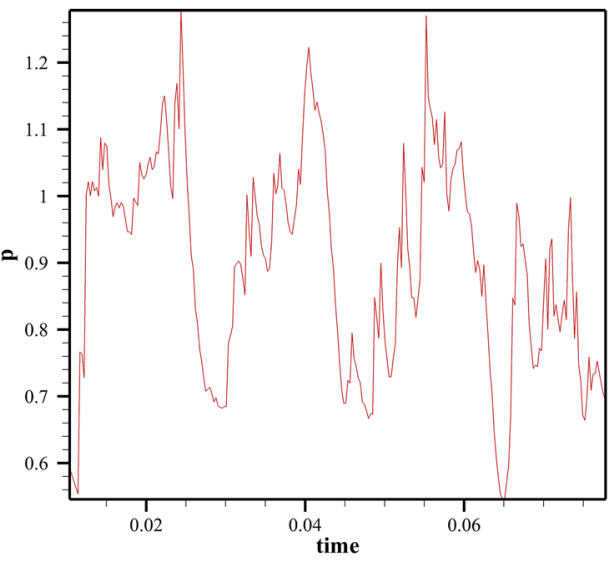

(d)
FIG. 17. Static pressure near the wall changes with time for different static pressure ratios (a) $p_{\text {iet }} / p_{\infty}=1.2$, (b) $p_{\text {jet }} / p_{\infty}=2.5$, (c) $p_{\text {jet }} / p_{\infty}=10$, and (d) $p_{\text {jet }} / p_{\infty}=30(\mathrm{DE} / \mathrm{CD}=2: 1)$. 


\section{ANALYSIS OF THE PERIOD OF THE SECONDARY JET}

\section{A. Pressure changes in the mixed zone}

The component calibration method is well suited to visualize the injection process of the secondary jet, but the process cannot be evaluated qualitatively. Therefore, other convenient and effective methods are needed to detect the secondary jets. We noticed that during the creation of the secondary jet, the secondary shock wave oscillates in the mixed zone. It is well known that the pressure before and after the shock wave differs. Due to the influences of the secondary shock wave on the pressure and the oscillatory motion of the secondary shock wave in the area in the mixed zone, we can easily quantify the process of injection of the secondary jet by detecting the pressure changes at certain points.

Five points are selected at a distance of 0.01 from the wall and the pressure changes are measured at these five points as shown in Fig. 14. The static pressure ratio of 2.5 is used as an example. The pressure changes at the five points are almost the same frequency, and they all exhibit 4 periods from $0.01 \mathrm{~s}$ to $0.1 \mathrm{~s}$ as shown in Fig. 15. This is in agreement with the results of the component calibration, where four periods were also detected during the same time. This proves that we can use pressure points to detect the secondary jet mathematically and accurately when the static pressure ratio is 2.5 .

However, the secondary shock wave is not the only reason for the pressure changes in the mixing zone. There are other reasons that cannot be ignored. After careful observation, we conclude that there are three reasons for the pressure changes in the mixed zone:

First, owing to the interaction of the jet shear layer and the supersonic incoming flow shear layer, the jet shear layer comprehensive shakes up and down and the secondary jet shoots into the mixed zone. As mentioned earlier, the secondary jet produces the secondary shock wave, which changes the pressure in the mixed zone.

Second, not only the jet shear layer comprehensively shakes up but also the jet shear layer shakes up partly. Because of the local perturbations of the jet shear layer, weak shock waves are produced, which may influence the pressure changes in the mixed zone.

Finally, the supersonic incoming flow shear layer is also an important factor affecting the pressure changes in the mixed zone. Our observations indicated that the supersonic incoming flow shear layer may become unstable early on. This instability leads to the deformation of the supersonic flow shear layer, which results in weak shock waves. These shock waves also affect the pressure changes in the mixed zone.
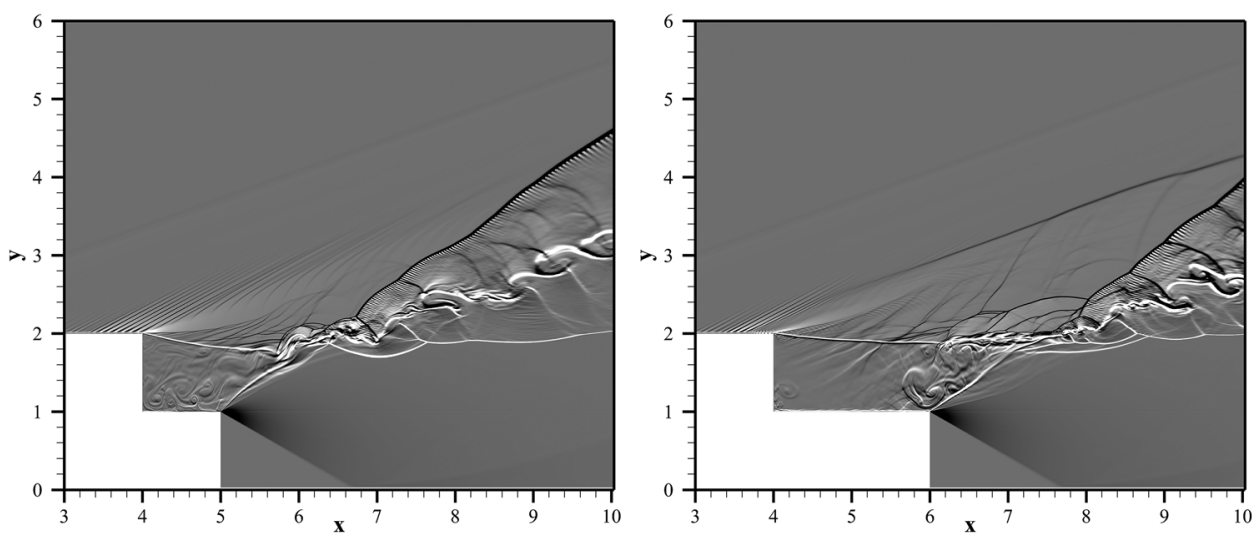

(a)

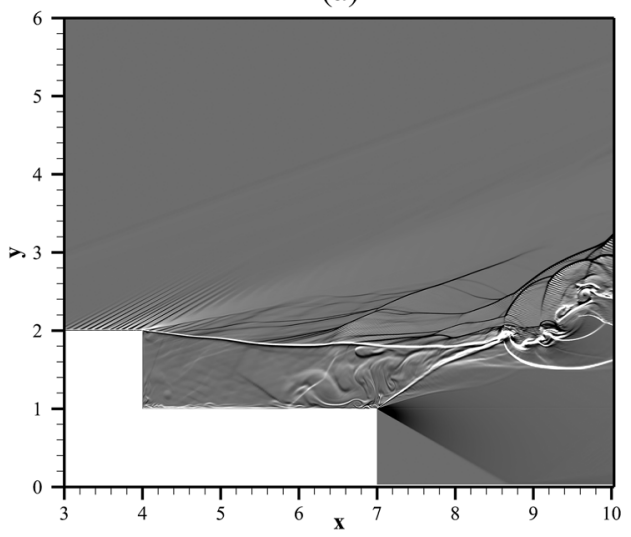

(c)

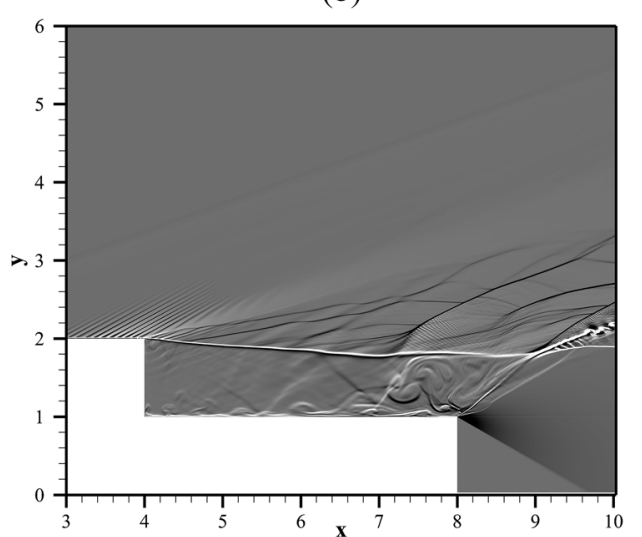

(d)
FIG. 18. Density gradients for different aspect ratios: (a) $D E / C D=1: 1$, (b) $D E / C D=2: 1$, (c) $D E / C D=3: 1$, and $(d)$ $D E / C D=4: 1\left(p_{\text {jet }} / p_{\infty}=10\right)$. 


\section{B. Different static pressure ratios}

In order to determine the influence of different static pressure ratios on the secondary jet, static pressure ratios of $1.2,2.5,10$, and 30 are used and the pressure changes are measured at the five points which are mentioned in Sec. IV A.

As shown in Fig. 16, the flow field structures of different static pressure ratios are similar.

However, as the static pressure ratio increases, the horizontal angle of the secondary jet increases significantly. In addition, the amount of material injected into the recirculation zone by the secondary jet is also significantly increased. The disturbance on the secondary jet is also significantly more, and more shocks can be observed in Fig. 16(d).

Four cycles are observed in the time period as shown in Fig. 17. The cycle is approximately $0.025 \mathrm{~s}$, and the frequency of the period is around $40 \mathrm{~Hz}$. This means that the frequency of the overall periodic fluctuation exhibits almost no change when the static pressure ratios increase. This occurs because the jet shear layer is partially perturbed. We observe that there are weak fluctuations in the main period. When the static pressure ratio is small, the overall periodic fluctuations of the jet shear layer are dominant. On the other hand, the effect of the weak periodic fluctuations of the jet shear layer is small but it exists. We did not observe the instability of the incoming shear layer. The supersonic incoming flow shear layer is stable under the two operating conditions. In the case of an under-expanded jet, the supersonic flow shear layer is stable if the static pressure ratio is small.

However, as Fig. 17(d) shows, when the static pressure ratio is 30, there are many fluctuations in each of the four cycles. Each of the four cycles lasts about $0.025 \mathrm{~s}$. These results indicate that even if the static pressure ratio changes, the period of the pressure point does not change. The comprehensive disturbance of the jet shear layer produces the main cycles. In addition, partial disturbances of the jet shear layer and the supersonic flow shear layer may cause destabilization and also lead to pressure changes in the mixed zone. As the static pressure ratio increases, the interaction between the jet shear layer and the supersonic incoming flow shear layer also becomes stronger. This increases the instability of the shear layer. When the static pressure ratio is large, the influences of the partial perturbations of the jet shear layer and the destabilization of the supersonic incoming flow shear layer are larger. Therefore, more fluctuations are observed in each cycle. However, we can still observe the complete cycles, as shown in Fig. 17(d). Even though the

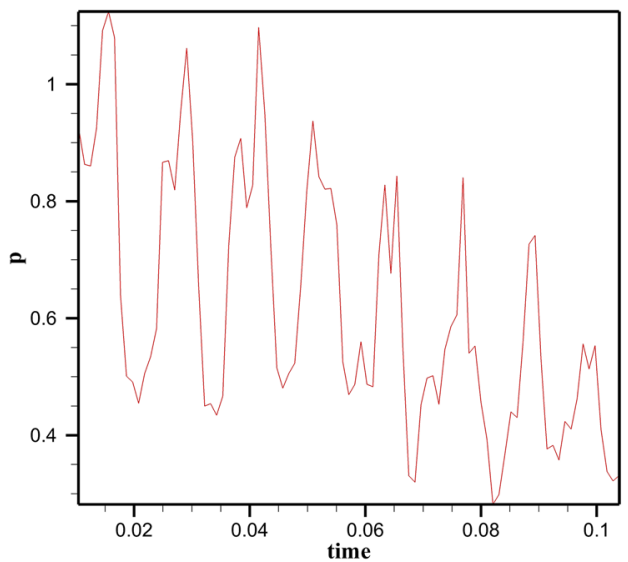

(a)

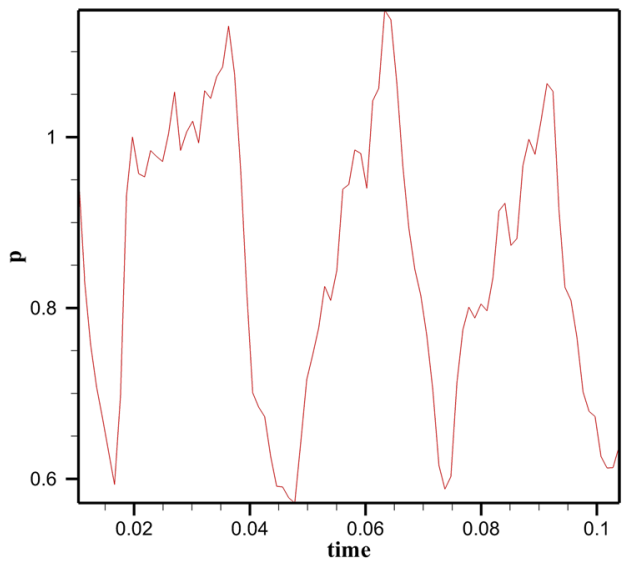

(c)

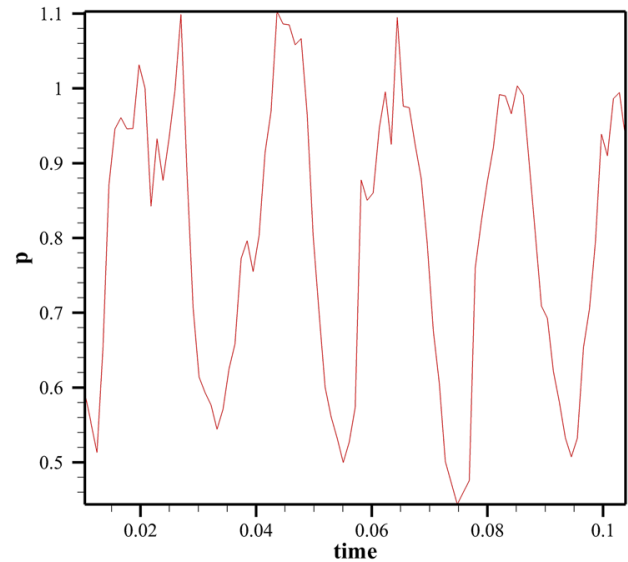

(b)

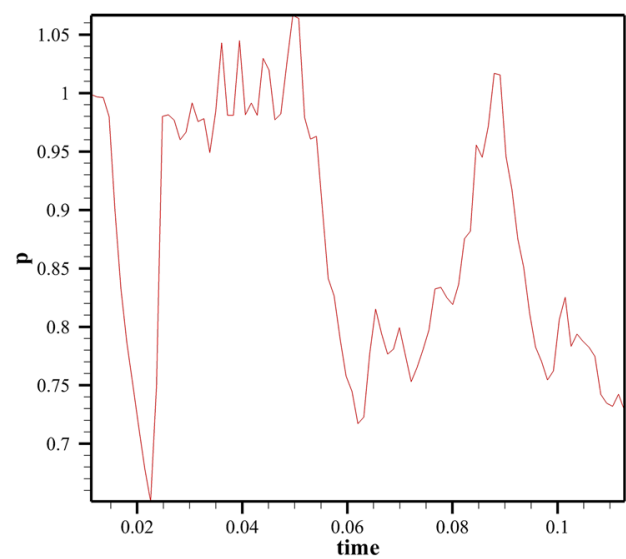

(d)
FIG. 19. Wall pressure distribution for different aspect ratios: (a) $D E / C D=1: 1$, (b) $\mathrm{DE} / \mathrm{CD}=2: 1$, (c) $\mathrm{DE} / \mathrm{CD}=3: 1$, and $(\mathrm{d})$ $\mathrm{DE} / \mathrm{CD}=4: 1\left(p_{\mathrm{jet}} / p_{\infty}=10\right)$ 
pressure ratio changes, the main cycle is still approximately $0.025 \mathrm{~s}$ and the frequency of the period is about $40 \mathrm{~Hz}$.

There is a little correlation between the periodicity of the secondary jet and the change in the static pressure ratio. However, a larger static pressure ratio results in greater instability of the shear layer, which, in turn, leads to a larger influence of the partial perturbation of the jet shear layer and the destabilization of the supersonic incoming flow shear layer. It is evident that there are more fluctuations in the four cycles at a static pressure ratio of 30 .

\section{Different aspect ratios of DE/CD}

The effects of the following aspect ratios are investigated: $\mathrm{DE} / \mathrm{CD}=1: 1,2: 1,3: 1$, and $4: 1$. The ratio of the jet's static pressure to the incoming flow's static pressure is 10 . Five pressure points are selected near CD and their changes are observed.

The density gradient map is shown in Fig. 18. The flow field structure is similar for the four conditions. When the DE/CD of the recirculation zone wall is $1: 1$, the recirculation zone is small and the injected secondary jet can directly interact with the wall surface. Under the other conditions, the secondary jet is not in direct contact with the wall.

As Fig. 19(a) shows, several cycles in the interval are observed. At $\mathrm{DE} / \mathrm{CD}=1: 1$, the cycle time is very short and ranges from $0.01 \mathrm{~s}$ to $0.015 \mathrm{~s}$. As the $\mathrm{DE} / \mathrm{CD}$ ratio increases, the cycle time increases. At $\mathrm{DE} / \mathrm{CD}=2: 1$, the cycle time is approximately $0.02 \mathrm{~s}$; at $\mathrm{DE} / \mathrm{CD}$ $=3: 1$, the cycle time is about $0.03 \mathrm{~s}$; and at $\mathrm{DE} / \mathrm{CD}=4: 1$, the cycle time is about $0.04 \mathrm{~s}$. This indicates that the frequency of the overall periodic fluctuations decreases with an increase in the static pressure ratios.

\section{CONCLUSION}

1. The interaction between the jet shear layer and the supersonic incoming flow shear layer results in the secondary jet flow and a mixed shear layer. Two weak shock waves occur on both sides of the mixed shear layer. Due to differences in the speeds on both sides of the mixed shear layer, the mixing shear layer is unstable and forms some vortex structures.

2. The secondary jet has unique periodicity. The periodicity is related to the overall oscillation of the shear layer. The pressure of the mixed zone is influenced by three factors, i.e., the comprehensive perturbation of the jet shear layer, the partial perturbation of the jet shear layer, and the instability of the supersonic shear layer. The comprehensive perturbation of the jet shear layer is the main factor affecting the pressure changes in the mixed zone. As the static pressure ratio increases, the effects of the other two factors intensify. This means that the increase in the pressure is caused by the increased interaction between the two shear layers.

3. When $\mathrm{DE} / \mathrm{CD}=2: 1$ and the static pressure ratio $p_{\text {jet }} / p_{\infty}$ is changed, the period of the secondary jet is almost the same of about $0.025 \mathrm{~s}$ and the frequency is about $40 \mathrm{~Hz}$. Thus, the period of the secondary jet does not change as the static pressure ratio changes. Different ratios of DE/CD in the wall area of the recirculation zone affect the period of the secondary jet. The larger the ratio, the weaker the effect of the two shear layers and the longer the period of the secondary jet are.

\section{ACKNOWLEDGMENTS}

This study was supported by the National Key Research and Development Program of China (Grant No. 2016YFA0401201) and the National Natural Science Foundation of China (Grant Nos. 11872066, 11472281, 11727901, and 11532014).

\section{APPENDIX: DISCRETE CONVECTION TERM}

The discrete scheme of the convective term is

$$
\operatorname{CONV}_{i, j}^{n}=\frac{\left(\tilde{\boldsymbol{F}}_{i+\frac{1}{2}, j}^{n}-\tilde{\boldsymbol{F}}_{i-\frac{1}{2}, j}^{n}\right)}{\Delta x}+\frac{\left(\tilde{\boldsymbol{G}}_{i, j+\frac{1}{2}}^{n}-\tilde{\boldsymbol{G}}_{i, j-\frac{1}{2}}^{n}\right)}{\Delta y} .
$$

The flux vectors are as follows:

$$
\left\{\begin{array}{l}
\tilde{\boldsymbol{F}}_{i+\frac{1}{2}, j}=\tilde{\boldsymbol{F}}_{i+\frac{1}{2}, j}^{+}+\tilde{\boldsymbol{F}}_{i+\frac{1}{2}, j}^{-} \\
\tilde{\boldsymbol{G}}_{i, j+\frac{1}{2}}=\tilde{\boldsymbol{G}}_{i, j+\frac{1}{2}}^{+}+\tilde{\boldsymbol{G}}_{i, j+\frac{1}{2}}^{-},
\end{array}\right.
$$

where

$$
\begin{aligned}
& \tilde{\boldsymbol{F}}_{i+\frac{1}{2}, j}^{ \pm}=\sum_{k=0}^{2} \omega_{x k}^{ \pm} q_{x k}^{ \pm}, \\
& \tilde{\boldsymbol{G}}_{i+\frac{1}{2}, j}^{ \pm}=\sum_{k=0}^{2} \omega_{y k}^{ \pm} q_{y k}^{ \pm} .
\end{aligned}
$$

The best weighting factor suggested by Jiang and $S h u^{15}$ is

$$
\omega_{x k}^{ \pm}=\frac{\alpha_{x k}^{ \pm}}{\alpha_{x 0}^{ \pm}+\alpha_{x 1}^{ \pm}+\alpha_{x 2}^{ \pm}}, \alpha_{x k}^{ \pm}=\frac{C_{x k}^{ \pm}}{\left(\varepsilon+I S_{x k}^{ \pm}\right)^{n}} \quad(k=0,1,2) .
$$

$p$ is an integer, usually greater than 2 , as suggested by Jiang and Shu.

The coefficients and formula can be shown as follows:

$$
\begin{gathered}
C_{x 0}^{+}=\frac{1}{10}, \quad C_{x 1}^{+}=\frac{3}{5}, \quad C_{x 2}^{+}=\frac{3}{10}, \\
C_{x 0}^{-}=\frac{3}{10}, \quad C_{x 1}^{-}=\frac{3}{5}, \quad C_{x 2}^{-}=\frac{1}{10}, \\
\left\{\begin{array}{l}
I S_{x 0}^{+}=\frac{13}{12}\left(\mathbf{F}_{i-2, j}^{+}-2 \mathbf{F}_{i-1, j}^{+}+\mathbf{F}_{i, j}^{+}\right)^{2}+\frac{1}{4}\left(\mathbf{F}_{i-2, j}^{+}-4 \mathbf{F}_{i-1, j}^{+}+3 \mathbf{F}_{i, j}^{+}\right)^{2}, \\
I S_{x 1}^{+}=\frac{13}{12}\left(\mathbf{F}_{i-1, j}^{+}-2 \mathbf{F}_{i, j}^{+}+\mathbf{F}_{i+1, j}^{+}\right)^{2}+\frac{1}{4}\left(\mathbf{F}_{i-1, j}^{+}-\mathbf{F}_{i+1, j}^{+}\right)^{2}, \\
I S_{x 2}^{+}=\frac{13}{12}\left(\mathbf{F}_{i, j}^{+}-2 \mathbf{F}_{i+1, j}^{+}+\mathbf{F}_{i+2, j}^{+}\right)^{2}+\frac{1}{4}\left(3 \mathbf{F}_{i, j}^{+}-4 \mathbf{F}_{i+1, j}^{+}+\mathbf{F}_{i+2, j}^{+}\right)^{2}, \\
I S_{x 0}^{-}=\frac{13}{12}\left(\mathbf{F}_{i, j}^{-}-2 \mathbf{F}_{i+1, j}^{-}+\mathbf{F}_{i+2, j}^{-}\right)^{2}+\frac{1}{4}\left(\mathbf{F}_{i, j}^{-}-\mathbf{F}_{i+2, j}^{-}\right)^{2}, \\
I S_{x 0}^{-}=\frac{13}{12}\left(\mathbf{F}_{i+1, j}^{-}-2 \mathbf{F}_{i+2, j}^{-}+\mathbf{F}_{i+3, j}^{-}\right)^{2}+\frac{1}{4}\left(3 \mathbf{F}_{i+1, j}^{-}-4 \mathbf{F}_{i+2, j}^{-}+\mathbf{F}_{i+3, j}^{-}\right)^{2}, \\
\left\{\begin{array}{l}
q_{x 0}^{+}=\frac{1}{3} \mathbf{F}_{i-2, j}^{+}-\frac{7}{6} \mathbf{F}_{i-1, j}^{+}+\frac{11}{6} \mathbf{F}_{i, j}^{+}, \\
q_{x 1}^{+}=-\frac{1}{6} \mathbf{F}_{i-1, j}^{+}+\frac{5}{6} \mathbf{F}_{i, j}^{+}+\frac{1}{3} \mathbf{F}_{i+1, j}^{+}, \\
q_{x 2}^{+}=\frac{1}{3} \mathbf{F}_{i, j}^{+}+\frac{5}{6} \mathbf{F}_{i+1, j}^{+}-\frac{1}{6} \mathbf{F}_{i+2, j}^{+},
\end{array} \mathbf{F}_{i+1, j}^{-}\right)^{2}+\frac{1}{4}\left(\mathbf{F}_{i-1, j}^{-}-4 \mathbf{F}_{i, j}^{-}+3 \mathbf{F}_{i+1, j}\right)^{2},
\end{array}\right.
\end{gathered}
$$




$$
\left\{\begin{array}{l}
q_{x 0}^{-}=-\frac{1}{6} \mathbf{F}_{i-1, j}^{-}+\frac{5}{6} \mathbf{F}_{i, j}^{-}+\frac{1}{3} \mathbf{F}_{i+1, j}^{-}, \\
q_{x 1}^{-}=\frac{1}{3} \mathbf{F}_{i, j}^{-}+\frac{5}{6} \mathbf{F}_{i+1, j}^{-}+\frac{1}{6} \mathbf{F}_{i+2, j}^{-}, \\
q_{x 2}^{-}=\frac{11}{6} \mathbf{F}_{i+1, j}^{-}-\frac{7}{6} \mathbf{F}_{i+2, j}^{-}+\frac{1}{3} \mathbf{F}_{i+3, j}^{-},
\end{array}\right.
$$

where $\mathbf{F}_{i, j}^{ \pm}$stands for the Steger-Warming flux vector splitting. Furthermore, the calculation formula for the other coordinate can be operated by symmetricity.

\section{REFERENCES}

${ }^{1} \mathrm{~W}$. Huang and L. Yan, "Numerical investigation on the ram-scram transition mechanism in a strut-based dual-mode scramjet combustor," Int. J. Hydrogen Energy 41(8), 4799-4807 (2016).

${ }^{2}$ J. E. Reardon and H. F. Nelson, "Rocket plume base heating methodology," J. Thermophys. Heat Transfer 8(2), 216 (1994).

${ }^{3}$ H. B. Ebrahimi, "Numerical investigation of jet interaction in a supersonic freestream,” J. Spacecr. Rockets 45(1), 95 (2008).

${ }^{4}$ J. Troyes, I. Dubois, V. Borie et al., "Multi-phase reactive numerical simulations of a model solid rocket exhaust jet," in 42nd AIAA/ ASME/SAE/ASEE Joint Propulsion Conference and Exhibit (AIAA, 2006), p. 4414.

${ }^{5}$ R. Michael, "Infrared signature generation of airborne targets," Proc. SPIE 1967, 114-122 (1993).
${ }^{6} \mathrm{X}$. Ma and A. Schröder, "Analysis of flapping motion of reattaching shear layer behind a two-dimensional backward-facing step," Phys. Fluids 29(11), 115104 (2017).

${ }^{7}$ H. Park, W. P. Jeon, H. Choi et al., "Mixing enhancement behind a backwardfacing step using tabs," Phys. Fluids 19(10), 105103 (2007).

${ }^{8}$ M. A. Kegerise, E. F. Spina, S. Garg et al., "Mode-switching and nonlinear effects in compressible flow over a cavity," Phys. Fluids 16(3), 678-687 (2004).

${ }^{9}$ T. W. H. Sheu and H. P. Rani, "Exploration of vortex dynamics for transitional flows in a three-dimensional backward-facing step channel," J. Fluid Mech. 550, 61-83 (2006).

${ }^{10}$ S. D. Hall, M. Behnia, C. A. J. Fletcher et al., "Investigation of the secondary corner vortex in a benchmark turbulent backward-facing step using cross-correlation particle imaging velocimetry," Exp. Fluids 35(2), 139-151 (2003).

${ }^{11} \mathrm{~F}$. Génin and S. Menon, "Studies of shock/turbulent shear layer interaction using large-eddy simulation," Comput. Fluids 39(5), 800-819 (2010).

${ }^{12}$ T. Suzuki and S. K. Lele, "Shock leakage through an unsteady vortex-laden mixing layer: Application to jet screech," J. Fluid Mech. 490, 139-167 (2003).

${ }^{13}$ J. M. Cohen and J. C. Bennett, "An experimental study of the transient flow over a backward-facing step," AIAA Paper No. 96-0322, 1996.

${ }^{14} \mathrm{P}$. D. Weidman and C. Y. Wang, "Boundary layers at the interface of two different shear flows," Phys. Fluids 30(5), 053604 (2018).

${ }^{15}$ G. S. Jiang and C. W. Shu, "Efficient implementation of weighted ENO schemes," J. Comput. Phys. 126(1), 202-228 (1996).

${ }^{16} \mathrm{D}$. Fu, Y. Ma, and X. Li, Direct Numerical Simulation of Compressible Turbulence (Science Press, 2010).

${ }^{17}$ J. R. Dormand and P. J. Prince, "A family of embedded Runge-Kutta formulae," J. Comput. Appl. Math. 6(1), 19-26 (1980). 